\title{
ON THE EIGENVALUES AND EIGENFUNCTIONS FOR A FREE BOUNDARY PROBLEM FOR INCOMPRESSIBLE VISCOUS MAGNETOHYDRODYNAMICS
}

Abstract. The motion of incompressible magnetohydrodynamics (mhd) in a domain bounded by a free surface and coupled through it with an external electromagnetic field is considered. Transmission conditions for electric currents and magnetic fields are prescribed on the free surface. In this paper we show the idea of the proof of local existence by the method of successive approximations. For this we need linearized problems: the Stokes system for the velocity and pressure and the linear transmission problem for the electromagnetic field. We do not prove the local existence of solutions to the original problem but we show existence of a fundamental basis of functions for the linearized problems. Once we have such a basis, the existence of solutions to the linear problems can be shown by the Faedo-Galerkin method, as in other papers of Kacprzyk. The existence of solutions of the linear systems can also be shown by the method of regularizer.

1. Introduction. We consider a free boundary problem for magnetohydrodynamic motions in a domain $\stackrel{1}{\Omega_{t}}$ interacting through a free surface $S_{t}$

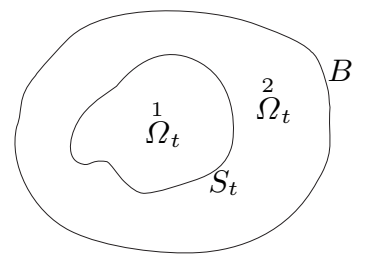

2010 Mathematics Subject Classification: 35A01, 35Q30, 35R35, 76D05, 76W05, 76X05. Key words and phrases: free boundary, incompresible magnetohydrodynamics, FaedoGalerkin method.

Received 30 November 2017; revised 19 July 2018.

Published online 17 June 2019. 
with an electromagnetic field located in $\stackrel{2}{\Omega}_{t}$. In $\stackrel{1}{\Omega}$ the magnetohydrodynamic motion is described by the system of equations

$$
\begin{aligned}
& v_{, t}+v \cdot \nabla v-\operatorname{div} \mathbb{T}(v, p)-\mu_{1}^{H} \cdot \nabla \stackrel{1}{H}+\frac{1}{2} \mu_{1} \nabla \stackrel{1}{H}^{2}=f, \\
& \operatorname{div} v=0, \\
& \stackrel{1}{H}_{, t}=-\operatorname{rot} \stackrel{1}{E}, \\
& \operatorname{rot} \stackrel{1}{H}=\sigma_{1}\left(\stackrel{1}{E}+\mu_{1} v \times \stackrel{1}{H}\right), \\
& \operatorname{div} \stackrel{1}{H}=0,
\end{aligned}
$$

where $v=v(x, t)=\left(v_{1}(x, t), v_{2}(x, t), v_{3}(x, t)\right) \in \mathbb{R}^{3}$ is the velocity of the fluid, $p=p(x, t) \in \mathbb{R}$ is the pressure, $\stackrel{1}{H}(x, t)=\left(\stackrel{1}{H}{ }_{1}(x, t), \stackrel{1}{H}{ }_{2}(x, t), \stackrel{1}{H}{ }_{3}(x, t)\right)$ $\in \mathbb{R}^{3}$ is the magnetic field, $\stackrel{1}{E}=\stackrel{1}{E}(x, t)=\left(\stackrel{1}{E}_{1}(x, t), \stackrel{1}{E_{2}}(x, t), \stackrel{1}{E_{3}}(x, t)\right) \in \mathbb{R}^{3}$ is the electric field, $f=f(x, t)=\left(f_{1}(x, t), f_{2}(x, t), f_{3}(x, t)\right) \in \mathbb{R}^{3}$ is the external force field per unit mass, and $x=\left(x_{1}, x_{2}, x_{3}\right)$ are the Cartesian coordinates. Moreover, $\mu_{1}$ is the constant magnetic permeability and $\sigma_{1}$ the constant electric conductivity. We denote by $\mathbb{T}(v, p)$ the stress tensor of the form

$$
\mathbb{T}(v, p)=\nu \mathbb{D}(v)-p I,
$$

where $\nu$ is a positive viscosity coefficient, $I$ is the unit matrix and $\mathbb{D}(v)$ is the dilatation tensor of the form

$$
\mathbb{D}(v)=\left\{v_{i, x_{j}}+v_{j, x_{i}}\right\}_{i, j=1,2,3} .
$$

For system 1.1 the following initial and boundary conditions are prescribed:

$$
\begin{aligned}
& \bar{n} \cdot \mathbb{T}(v, p)+\mu_{1} \bar{n} \cdot \mathbb{T}(\stackrel{1}{H})=p_{0} \bar{n} \quad \text { on } S_{t}, \\
& \left.v\right|_{t=0}=v(0),\left.\quad \stackrel{1}{H}\right|_{t=0}=\stackrel{1}{H}(0), \\
& \left.\stackrel{1}{\Omega_{t}}\right|_{t=0}=\stackrel{1}{\Omega_{0}},\left.\quad S_{t}\right|_{t=0}=S_{0},
\end{aligned}
$$

where $\bar{n}$ is the unit vector outward to $\stackrel{1}{\Omega}_{t}$ and normal to $S_{t}$, and

$$
\mathbb{T}(\stackrel{1}{H})=\left\{\stackrel{1}{H}_{i} \stackrel{1}{H}_{j}-\frac{1}{2} \stackrel{1}{H}^{2} \delta_{i j}\right\}_{i, j=1,2,3} .
$$

Finally, $1.4{ }_{1}$ implies the compatibility conditions

$$
\begin{aligned}
& \bar{n}_{0} \cdot \mathbb{D}(v(0)) \cdot \bar{\tau}_{\alpha 0}+\mu_{1} \bar{n}_{0} \cdot \stackrel{1}{H}(0) \bar{\tau}_{\alpha 0} \cdot \stackrel{1}{H}(0)=0, \\
& \bar{n}_{0}=\left.\bar{n}\right|_{t=0}, \quad \bar{\tau}_{\alpha 0}=\left.\bar{\tau}_{\alpha}\right|_{t=0}, \quad \alpha=1,2 .
\end{aligned}
$$


In $\stackrel{2}{\Omega_{t}}$ we have a motionless dielectric gas under constant pressure $p_{0}$. Therefore, we only have an electromagnetic field described by the system

$$
\begin{aligned}
& \stackrel{2}{H}_{, t}=-\operatorname{rot} \stackrel{2}{E}, \\
& \sigma_{2} \stackrel{2}{E}=\operatorname{rot} \stackrel{2}{H}, \\
& \operatorname{div} \stackrel{2}{H}=0 .
\end{aligned}
$$

For system (1.6) the following initial and boundary conditions are prescribed:

$$
\begin{aligned}
& \left.\stackrel{2}{H}\right|_{t=0}=\stackrel{2}{H}(0),\left.\left.\quad \stackrel{2}{\Omega}\right|_{t}\right|_{t=0}=\stackrel{2}{\Omega_{0}}, \\
& \left.\stackrel{2}{H}\right|_{B}=0 .
\end{aligned}
$$

The homogeneous boundary condition on $B$ is assumed for simplicity only. We can prescribe here either a magnetic field or an electric current.

Electromagnetic fields in $\stackrel{1}{\Omega}$ t and $\stackrel{2}{\Omega}$ t are coupled through $S_{t}$ via the following transmission conditions:

$$
\begin{aligned}
& \stackrel{1}{E} \cdot \bar{\tau}_{\alpha}=\left.\stackrel{2}{E} \cdot \bar{\tau}_{\alpha}\right|_{S_{t}}, \\
& \bar{n} \times \bar{\tau}_{\alpha} \cdot \stackrel{1}{H}=\bar{n} \times\left.\bar{\tau}_{\alpha} \cdot \stackrel{2}{H}\right|_{S_{t}}, \quad \alpha=1,2, \\
& \stackrel{1}{H} \cdot \bar{n}=\mu_{2} \stackrel{2}{H} \cdot \bar{n},
\end{aligned}
$$

where $\bar{\tau}_{1}, \bar{\tau}_{2}, \bar{n}$ is an orthonormal system of vectors in a neighborhood of $S_{t}$ such that $\left.\bar{n}\right|_{S_{t}}$ is normal to $S_{t}$ and $\bar{\tau}_{1},\left.\bar{\tau}_{2}\right|_{S_{t}}$ are tangent to $S_{t}$.

Now we explain the physical meaning of the transmission conditions (1.8). The currents are defined by $j_{1}=\sigma_{1}(\stackrel{1}{E}+v \times \stackrel{1}{H}), j_{2}=\sigma_{2} \stackrel{2}{E}$. Therefore 1.8$)_{1}$ means that the jumps of the tangent components of the currents are described by

$$
\frac{1}{\sigma_{1}} j_{1} \cdot \bar{\tau}_{\alpha}-v \times \stackrel{1}{H} \cdot \bar{\tau}_{\alpha}=\frac{1}{\sigma_{1}} j_{2} \cdot \bar{\tau}_{\alpha}, \quad \alpha=1,2, \quad \text { on } S_{t},
$$

for $\sigma_{1} \neq \sigma_{2}$. Conditions $(1.8)_{2}$ mean that the tangent components of the magnetic field are continuous through $S_{t}$. To describe $(1.8) 3$ we recall the magnetic induction $\stackrel{i}{B}=\mu_{i} \stackrel{i}{H}, i=1$, 2 . Since $\operatorname{div} \stackrel{i}{B}=0$ in $\stackrel{i}{\Omega}$, we derive $(1.8)_{3}$. It means that the normal component of the magnetic induction is continuous on $S_{t}$. Hence there is no jump of the magnetic induction flux.

To prove existence of solutions to problem (1.1)-(1.8) we transform it into two problems: a problem for the fluid motion and a problem for the 
electromagnetic field. Therefore, for given $\stackrel{1}{H}$ we have the problem for $(v, p)$ :

$$
\begin{array}{lr}
v_{t}+v \cdot \nabla v-\operatorname{div} \mathbb{T}(v, p)=f+\mu_{1} \operatorname{div} \mathbb{T}(\stackrel{1}{H}) & \text { in } \Omega_{t}, \\
\operatorname{div} v=0 & \text { in } \Omega_{t}, \\
\bar{n} \cdot \mathbb{T}(v, p)=p_{0} \bar{n}-\mu_{1} \bar{n} \cdot \mathbb{T}(\stackrel{1}{H}) & \text { on } S_{t}, \\
\left.v\right|_{t=0}=v(0) & \text { in } \Omega_{0} .
\end{array}
$$

Next for given $v$, the electromagnetic field is determined by the problem

$$
\begin{aligned}
& \mu_{1} \stackrel{1}{H}_{, t}=-\operatorname{rot} \stackrel{1}{E}, \quad \operatorname{rot} \stackrel{1}{H}=\sigma_{1}\left(\stackrel{1}{E}+\mu_{1} v \times \stackrel{1}{H}\right) \quad \text { in } \stackrel{1}{\Omega}, \\
& \mu_{2} \stackrel{2}{H}_{, t}=-\operatorname{rot} \stackrel{2}{E}, \quad \sigma_{2} \stackrel{2}{E}=\operatorname{rot} \stackrel{2}{H} \quad \text { in } \stackrel{2}{\Omega_{t}}, \\
& \left.\stackrel{1}{H}\right|_{t=0}=\stackrel{1}{H}(0), \quad \operatorname{div} \stackrel{1}{H}(0)=0 \quad \text { in } \stackrel{1}{\Omega_{0}}, \\
& \left.\stackrel{2}{H}\right|_{t=0}=\stackrel{2}{H}(0), \quad \operatorname{div} \stackrel{2}{H}(0)=0 \quad \text { in } \stackrel{2}{\Omega_{0}}, \\
& \left.\stackrel{2}{H}\right|_{B}=0 \text {, } \\
& \stackrel{1}{E} \cdot \bar{\tau}_{\alpha}=\stackrel{2}{E} \cdot \bar{\tau}_{\alpha}, \quad \bar{n} \times \bar{\tau}_{\alpha} \cdot \stackrel{1}{H}=\bar{n} \times \bar{\tau}_{\alpha} \cdot \stackrel{2}{H}, \quad \alpha=1,2, \\
& \mu_{1} \stackrel{1}{H} \cdot \bar{n}=\mu_{2} \stackrel{2}{H} \cdot \bar{n} \\
& \text { on } S_{t} \text {. }
\end{aligned}
$$

Since $(1.9)$ and $(1.10)$ are free boundary problems, the natural way to treat them is to pass to Lagrangian coordinates (see $|26|) . \stackrel{1}{\Omega} t, \stackrel{2}{\Omega_{t}}$ and $S_{t}$ are determined by the velocity $v$ of the fluid. However, equations 1.10 are not in the form appropriate for using Lagrangian coordinates. Moreover, in $\stackrel{2}{\Omega}_{t}$ there is no motion, so there is no velocity guaranteeing existence of Lagrangian coordinates.

Therefore, we construct an artificial velocity $\stackrel{2}{v}$ in $\stackrel{2}{\Omega_{t}}$ as a solution to problem (3.2). Moreover, for the field equations 1.10$)_{1,2}$,

$$
\mu_{i} \stackrel{i}{H}_{t}=-\operatorname{rot} \stackrel{i}{E}, \quad i=1,2,
$$

we add the term $\mu_{i}^{i} \cdot \vec{i} \cdot \stackrel{i}{H}, i=1,2$, to both sides of 1.11 , so we have

$$
\mu_{i}\left(\stackrel{i}{H}_{t}+\stackrel{i}{v} \cdot \nabla \stackrel{i}{H}\right)=-\operatorname{rot} \stackrel{i}{E}+\mu_{i} \cdot \stackrel{i}{v} \cdot \nabla \stackrel{i}{H}, \quad i=1,2 .
$$

These equations can be rewritten in Lagrangian coordinates and the term on the r.h.s. of 1.12 is of a lower order. Therefore the natural way to show existence of solutions to $1.9,1.10$ is the method of successive approximations described in Section 3 , 
In this paper we are not going to prove existence of solutions to problem (1.9), 1.10) (the result is shown in [5, 20, 21]). Our aim is to justify the energy method used in [5, 6] to prove existence of solutions to problem (1.9), (1.10). The existence is proved by applying the Faedo-Galerkin method to problems obtained by linearizing (1.9), 1.10 and formulated in fixed initial domains. Problems (3.7) and 3.13) are exactly such problems. In Sections 4 and 5 the existence of fundamental bases necessary for applying the FaedoGalerkin method is shown.

Now we present our results on existence of eigenfuctions and eigenvalues for problems (3.7) and (3.13). In the case of problem (3.7) we examine the elliptic problem (see (5.7))

$$
\begin{aligned}
& \stackrel{i}{\mu_{i} \psi}+\frac{1}{\sigma_{i}} \operatorname{rot}^{2} \stackrel{i}{\psi}=\lambda \stackrel{i}{\psi}+\stackrel{i}{f}, \quad \text { in } \stackrel{i}{\Omega_{0}}, i=1,2, \\
& \frac{1}{\sigma_{1}} \operatorname{rot} \stackrel{1}{\psi} \cdot \bar{\tau}_{\alpha}=\frac{1}{\sigma_{2}} \operatorname{rot} \stackrel{2}{\psi} \cdot \bar{\tau}_{\alpha}+g_{\alpha}, \quad \alpha=1,2, \quad \text { on } S_{0}, \\
& \stackrel{1}{\psi} \cdot \bar{n} \times \bar{\tau}_{\alpha}=\stackrel{2}{\psi} \cdot \bar{n} \times \bar{\tau}_{\alpha}, \quad \alpha=1,2, \quad \text { on } S_{0}, \\
& \stackrel{1}{\nu_{1} \psi \cdot \bar{n}}=\stackrel{2}{2} \nu_{2} \psi \cdot \bar{n} \quad \text { on } S_{0}, \\
& 2 \\
& \left.\psi\right|_{B}=0,
\end{aligned}
$$

and for problem (3.13) we consider the elliptic problem

$$
\begin{array}{ll}
\nu \varphi-\operatorname{div} \mathbb{T}(\varphi, q)=\lambda \varphi+f & \text { in } \Omega_{0}, \\
\operatorname{div} \varphi=g & \text { in } \Omega_{0}, \\
\bar{n} \cdot \mathbb{T}(\varphi, q)=k & \text { on } S_{0},
\end{array}
$$

where $\lambda$ are eigenvalues and $\nu, \mu_{1}, \mu_{2}$ are positive numbers.

THEOREM 1.1. There exist eigenfunctions and eigenvalues for problems 1.13 and 1.14 .

Proof. The existence of eigenfunctions and eigenvalues for problem 1.14 is proved by Temam [25]. The results for problem (1.13) are proved in Section 5 .

REMARK 1.2. In this paper we generalize the approach to problem 1.13 presented in [10] because more advanced techniques are used. Thanks to this, $L_{p}$-approach can be applied.

The paper is organized as follows. In Section 2 we introduce notation and some auxiliary results. In Section 3 the method of successive approximations is formulated. In Sections 4 and 5 the existence of fundamental bases to problems (3.13) and (3.7) respectively is proved. 
The existence of a fundamental basis for the Faedo-Galerkin method for problem (3.13) is discussed in Definition 4.1 and for problem (5.7) in Lemma 5.1. Having existence of weak solutions to the linearized problems by the Faedo-Galerkin method, the existence of solutions to nonlinear problems 1.9 , 1.10 is proved by the method of successive approximations described in [10, Section 3]. Global existence is proved in [6] for sufficiently small initial data. The existence of local solutions to $(1.9), 1.10)$ is proved in [5] by the energy method. To examine problem (1.10) it is necessary to use the transmission conditions (1.8). In Lemma 2.4 the transmission conditions $(1.8)$ are generalized to the form $(2.8)$ and then the fundamental energy identity takes the form 2.9). The transmission conditions (2.8) imply relations between the tangent components of the magnetic fields. To show Lemma 5.1, we also need a transmission condition for the normal component of the magnetic fields (see $(5.7)_{5}$ ). To relax condition $(5.7)_{5}$ we show that equations 2.10), 2.11) are invariant with respect to the homothetic transformation

$$
\stackrel{i}{H^{\prime}}=b_{i} \stackrel{i}{H}, \quad \stackrel{i}{E^{\prime}}=b_{i} \stackrel{i}{E}, \quad i=1,2,
$$

where $b_{i}, i=1,2$, are constants.

Then the transmission condition 2.8 for the tangent components of the magnetic fields and the homothetic transformation (1.15) imply that the following transmission condition for the magnetic fields is admissible:

$$
\alpha_{1} \stackrel{1}{H}=\alpha_{2} \stackrel{2}{H} \quad \text { on } S_{t},
$$

where $\alpha_{1}, \alpha_{2}$ are arbitrary constants.

The transmission condition 1.16 in the case $\alpha_{1}=\alpha_{2}=1$ strongly simplifies the proof of local and global estimates in [5, 6]. However, condition (1.16) implies jumps of the tangent components of the electric field which are described by $(1.15)_{2}$ and $(2.8)_{1}$. But 1.16$)$ is important to derive in the energy equality 2.16).

We have to emphasize that this paper plays a fundamental role in the proof of local existence of solutions to problem (1.1) $-(1.8)$ in [5]. The idea of the proof in [5] is based on the following steps. First, by linearization described in Section 3, the existence of weak solutions is proved by the Faedo-Galerkin method, so the existence of a fundamental basis proved in this paper is crucial. Next by the standard technique the regularity of weak solutions is increased. Finally, the existence of local solutions by the method of successive approximations is proved.

However, the Maxwell equations form a symmetric hyperbolic system whose analytical treatment is difficult because the equations are expressed in terms of rotation operators. Bykhovskiı̌ [1] derived many analytical re- 
sults for solutions to elliptic rot-div systems. Magnetohydrodynamics (mhd) couples Navier-Stokes equations with the Maxwell equations, neglecting the displacement currents and taking into account electrical conduction. The mhd equations can be found in [2] and [16, Ch. 8].

The first result on solvability of a transmission problem for the mhd system was proved by Ladyzhenskaya and Solonnikov [14]. In that paper fixed domains were considered. The first results on existence of solutions to problem (1.1)-1.10 were shown by Kacprzyk [7 9]. Free boundary problems for the mhd system were also considered by Padula-Solonnikov [18], FrolovaSolonnikov [4] and Frolova [3].

In those papers the external magnetic field satisfies the elliptic system

$$
\operatorname{rot} \stackrel{2}{H}=0, \quad \operatorname{div} \stackrel{2}{H}=0 .
$$

However, the transmission condition (1.4) in [3, 4] is different because it contains the surface tension.

Hence on the one hand the problem considered in $[3,4,18]$ is simpler than problem (1.1)-1.10, but on the other hand it is more complicated because the surface tension is taken into account.

Moreover, in [3, 4, 18 the passage from a free boundary problem to a problem with fixed boundary is made by using the Hanzawa transformation. This is essential difference from [5 9], where Lagrangian coordinates were used.

Finally, in 20, 21] problem (1.1)-1.10 was considered by ShibataZajączkowski using the $L_{p}$-approach and Lagrangian coordinates.

We use elements of vector and tensor calculus taken from [12. It is important to mention that the transmission conditions on $S_{t}$ appeared already in [14, 19]. The first result on the existence of solutions to a free boundary problem for the compressible Navier-Stokes equations was proved in [26] by the energy method. The ideas and techniques of this paper were used by Kacprzyk in [5].

2. Notation and auxiliary results. We do not distinguish in notation between norms of scalar and vector-valued functions. Let $\omega$ be a vector, $\omega=\left(\omega_{1}, \ldots, \omega_{n}\right)$. Then

$$
|\omega|=\left(\sum_{i=1}^{n}\left|\omega_{i}\right|^{2}\right)^{1 / 2} .
$$

Let $L_{p}(\Omega)=\left\{u: \int_{\Omega}|u|^{p} d x<\infty\right\}$ for $p \in[1, \infty]$.

We denote by $V_{2}^{0}\left(\Omega^{T}\right)$ the space of functions with the finite norm

$$
\|u\|_{V_{2}^{0}\left(\Omega^{T}\right)}=\|u\|_{L_{\infty}\left(0, T ; L_{2}(\Omega)\right)}+\|u\|_{L_{2}\left(0, T ; H^{1}(\Omega)\right)} .
$$

We shall use the notation $H^{l}(\Omega)=\left\{u: \sum_{|\alpha| \leq l}\left\|D_{x}^{\alpha} u\right\|_{L_{2}(\Omega)}<\infty\right\}$, where 
$D_{x}^{\alpha}=\partial_{x_{1}}^{\alpha_{1}} \partial_{x_{2}}^{\alpha_{2}} \partial_{x_{3}}^{\alpha_{3}},|\alpha|=\alpha_{1}+\alpha_{2}+\alpha_{3}, \alpha_{i} \in \mathbb{N}_{0}, i=1,2,3$, and $\mathbb{N}_{0}=\mathbb{N} \cup\{0\}$, $\Omega \subset \mathbb{R}^{3}$.

In Section 5 we also use the simplified notation

$$
\|u\|_{L_{p}(\Omega)}=|u|_{p, \Omega}, \quad\|u\|_{H^{s}(\Omega)}=\|u\|_{s, \Omega} .
$$

We denote by $c$ a generic constant which changes its value from formula to formula. Similarly we denote by $\varphi$ a generic function which is always positive and increasing.

Next we introduce a partition of unity. We define two collections $\left\{\omega^{(k)}\right\}$ and $\left\{\Omega^{(k)}\right\}, k \in \mathfrak{M} \cup \mathfrak{N}$, of open sets such that $\bar{\omega}^{(k)} \subset \Omega^{(k)} \subset \Omega_{0}=\stackrel{1}{\Omega_{0}} \cup \stackrel{2}{\Omega_{0}}$, $\bigcup_{k} \omega^{(k)}=\bigcup_{k} \Omega^{(k)}=\Omega_{0}, \bar{\Omega}^{(k)} \cap S_{0}=\emptyset$ for $k \in \mathfrak{M}=\mathfrak{M}_{1} \cup \mathfrak{M}_{2}, \bar{\Omega}^{(k)} \cap S_{0} \neq \emptyset$ for $k \in \mathfrak{N}_{1}$ and $\bar{\Omega}^{(k)} \cap B \neq \emptyset$ for $k \in \mathfrak{N}_{2}, \mathfrak{N}=\mathfrak{N}_{1} \cup \mathfrak{N}_{2}$. Moreover, subdomains with index $k \in \mathfrak{M}_{i}$ are included in $\stackrel{i}{\Omega}, i=1,2$. We assume that at most $N_{0}$ of the $\Omega^{(k)}$ have nonempty intersection, and $\sup _{k} \operatorname{diam} \Omega^{(k)} \leq 2 \lambda$, $\sup _{k} \operatorname{diam} \omega^{(k)} \leq \lambda$ for some $\lambda>0$. Let $\zeta^{(k)}(x)$ be a smooth function such that $0 \leq \zeta^{(k)}(x) \leq 1, \zeta^{(k)}(x)=1$ for $x \in \omega^{(k)}, \zeta^{(k)}(x)=0$ for $x \in \Omega_{0} \backslash \Omega^{(k)}$ and $\left|D_{x}^{\nu} \zeta^{(k)}(x)\right| \leq c / \lambda^{|\nu|}$. Then $1 \leq \sum_{k}\left(\zeta^{(k)}(x)\right)^{2} \leq N_{0}$. Introducing the function

$$
\eta^{(k)}(x)=\frac{\zeta^{(k)}(x)}{\sum_{l}\left(\zeta^{(l)}(x)\right)^{2}}
$$

we have $\eta^{(k)}(x)=0$ for $x \in \Omega_{0} \backslash \Omega^{(k)}, \sum_{k} \eta^{(k)}(x) \zeta^{(k)}(x)=1$ and $\left|D_{x}^{\nu} \eta^{(k)}(x)\right| \leq$ $c / \lambda^{|\nu|}$. We denote by $\xi^{(k)}$ the "center" of $\omega^{(k)}$ and $\Omega^{(k)}$ for $k \in \mathfrak{M}$ and the center of $\bar{\omega}^{(k)} \cap S_{0}$ and $\bar{\Omega}^{(k)} \cap S_{0}$ for $k \in \mathfrak{N}_{1}$ and the center of $\bar{\omega}^{(k)} \cap B$ and $\bar{\Omega}^{(k)} \cap B$ for $k \in \mathfrak{N}_{2}$. For $k \in \mathfrak{M}_{i}, \xi^{(k)} \in \stackrel{i}{\Omega}_{0}, i=1,2$. Let $x=\left(x_{1}, x_{2}, x_{3}\right)$ be the Cartesian system of coordinates with origin at the center of $\Omega$. Then by translations and rotations we introduce a local coordinate system $y=$ $\left(y_{1}, y_{2}, y_{3}\right)$ centered at $\xi^{(k)}, k \in \mathfrak{N}_{1}$, such that the part $\tilde{S}^{(k)}=S_{0} \cap \bar{\Omega}^{(k)}$ of the boundary $S_{0}$ is described by $y_{3}=F_{k}\left(y_{1}, y_{2}\right)$. We denote the transformation by $y=Y_{k}(x)$. Then we define new coordinates by

$$
z_{i}=y_{i}, \quad i=1,2, \quad z_{3}=y_{3}-F_{k}\left(y_{1}, y_{2}\right) .
$$

We will denote this transformation by $\hat{\Omega}^{(k)} \supset \hat{\omega}^{(k)} \ni z=\Phi_{k}(y)$ where $y \in \omega^{(k)} \subset \Omega^{(k)}$. We assume that the sets $\hat{\omega}^{(k)}, \hat{\Omega}^{(k)}$ are described in local coordinates near $\xi^{(k)}$ by the inequalities

$$
\begin{array}{ll}
\left|y_{i}\right|<\lambda, & i=1,2, \quad 0<y_{3}-F\left(y_{1}, y_{2}\right)<\lambda, \\
\left|y_{i}\right|<2 \lambda, & i=1,2, \quad 0<y_{3}-F\left(y_{1}, y_{2}\right)<2 \lambda,
\end{array}
$$

respectively. Let $\Psi_{k}=\Phi_{k} \circ Y_{k}$. Then $z=\Psi_{k}(x)$ and

$$
\hat{u}^{(k)}(z, t)=u\left(\Psi_{k}^{-1}(z), t\right), \quad \tilde{u}^{(k)}(z, t)=\hat{u}^{(k)}(z, t) \hat{\zeta}^{(k)}(z) .
$$


For $k \in \mathfrak{M}$ we have

$$
\tilde{u}^{(k)}(x, t)=u^{(k)}(x, t) \zeta^{(k)}(x) .
$$

To examine free boundary problems in hydrodynamics we use Lagrangian coordinates which are the initial data to the Cauchy problem

$$
\frac{d x}{d t}=v(x, t),\left.\quad x\right|_{t=0}=\xi \in \stackrel{1}{\Omega_{0}} .
$$

Therefore,

$$
x=x_{v}(\xi, t) \equiv \xi+\int_{0}^{t} \bar{v}(\xi, s) d s,
$$

where $\bar{v}(\xi, t)=v\left(x_{v}(\xi, t), t\right)$. To define Lagrangian coordinates in $\stackrel{2}{\Omega} t$ we need

Lemma 2.1 (see $[23 \mid)$. Let $X\left(\stackrel{1}{\Omega_{t}}\right)$ be some Sobolev space. Let $v \in X\left(\stackrel{1}{\Omega_{t}}\right)$ be divergence free. Then there exists an extension $v^{\prime}$ of $v$ on $\stackrel{1}{\Omega_{t}} \cup \stackrel{2}{\Omega_{t}}$ such that $v^{\prime}$ is divergence free, $\left.v^{\prime}\right|_{\Omega_{t}}=v$ and there exists a constant $c$ such that

$$
\left\|v^{\prime}\right\|_{X\left(\Omega_{t} \cup \Omega_{t}\right)} \leq c\|v\|_{X\left(\Omega_{t}\right)} .
$$

Applying Lemma 2.3 below to problem 3.2 we will describe the extension more precisely. In view of the definition of Lagrangian coordinates we have

$$
\begin{aligned}
& \stackrel{1}{\Omega_{t}}=\left\{x \in \mathbb{R}^{3}: x=x_{v}(\xi, t), \xi \in \stackrel{1}{\Omega_{0}}\right\}, \\
& S_{t}=\left\{x \in \mathbb{R}^{3}: x=x_{v}(\xi, t), \xi \in S_{0}\right\}, \\
& \stackrel{1}{\Omega_{t}} \cup \stackrel{2}{\Omega}_{t}=\left\{x \in \mathbb{R}^{3}: x=x_{v^{\prime}}(\xi, t), \xi \in \stackrel{1}{\Omega}_{0} \cup \stackrel{2}{\Omega}_{0}\right\} .
\end{aligned}
$$

To formulate our problem in Lagrangian coordinates we need the notation

$$
\begin{array}{ll}
\nabla_{\bar{v}}=\frac{\partial \xi_{k}}{\partial x} \frac{\partial}{\partial \xi_{k}}, & \mathbb{D}_{\bar{v}} \bar{u}=\nabla_{\bar{v}} \bar{u}+\left(\nabla_{\bar{v}} \bar{u}\right)^{T}, \\
\mathbb{T}_{\bar{v}}(\bar{u}, \bar{p})=\mathbb{D}_{\bar{v}}(\bar{u})-\bar{p} \mathbb{I}, & \operatorname{div}_{\bar{v}} \bar{v}=\partial_{x_{i}} \xi_{k} \partial_{\xi_{k}} \bar{v}_{i}=\nabla_{\bar{v}} \cdot \bar{v},
\end{array}
$$

where summation over repeated indices is assumed, and $\xi=\xi(x, t)$ is the inverse transformation to $x=x_{\bar{v}}(\xi, t)$. From [24], 26] we have

LemMA 2.2. Let $\Omega \subset \mathbb{R}^{3}$ be a given bounded domain. Let $v \in L_{2}(\Omega)$ be such that

$$
E_{\Omega}(v)=\int_{\Omega}\left(v_{j, x_{i}}+v_{i, x_{j}}\right)^{2} d x<\infty .
$$

Then there exists a constant $c$ such that

$$
\|v\|_{H^{1}(\Omega)}^{2} \leq c\left(E_{\Omega}(v)+\|v\|_{L_{2}(\Omega)}^{2}\right) .
$$


Let us consider the Stokes problem in a bounded domain $\Omega \subset \mathbb{R}^{3}$ with boundary $S$ :

$$
\begin{array}{ll}
\omega_{, t}-\operatorname{div} \mathbb{T}(\omega, q)=f & \text { in } \Omega^{T}=\Omega \times(0, T), \\
\operatorname{div} \omega=0 & \text { in } \Omega^{T}, \\
\omega=b & \text { on } S^{T}=S \times(0, T), \\
\left.\omega\right|_{t=0}=\omega_{0} & \text { in } \Omega .
\end{array}
$$

Lemma 2.3 (see 23$]$ ). Assume that $f \in L_{p}\left(\Omega^{T}\right), b \in W_{p}^{2-1 / p, 1-1 / 2 p}\left(S^{T}\right)$, $\omega_{0} \in W_{p}^{2-2 / p}(\Omega), p \in(1, \infty), S \in C^{2}$. Then there exists a solution to problem (2.6) such that $\omega \in W_{p}^{2,1}\left(\Omega^{T}\right), \nabla q \in L_{p}\left(\Omega^{T}\right)$, and we have the estimate

$$
\begin{aligned}
& \|\omega\|_{W_{p}^{2,1}\left(\Omega^{T}\right)}+\|\nabla q\|_{L_{p}\left(\Omega^{T}\right)} \\
& \quad \leq c\left(\|f\|_{L_{p}\left(\Omega^{T}\right)}+\|b\|_{W_{p}^{2-1 / p, 1-1 / 2 p}\left(S^{T}\right)}+\left\|\omega_{0}\right\|_{W_{p}^{2-2 / p}(\Omega)}\right) .
\end{aligned}
$$

Now we justify the transmission condition from 1.10 . need

To obtain the energy type estimates for solutions to problem 1.10 we

Lemma 2.4. Assume the following transmission conditions on $S_{t}$ :

$$
a_{1}^{\nu_{1}} \stackrel{1}{E} \cdot \bar{\tau}_{\alpha}=a_{2}^{\nu_{1}} \stackrel{2}{E} \cdot \bar{\tau}_{\alpha}, \quad a_{1}^{\nu_{2}} \bar{n} \times \bar{\tau}_{\alpha} \cdot \stackrel{1}{H}=a_{2}^{\nu_{2}} \bar{n} \times \bar{\tau}_{\alpha} \cdot \stackrel{2}{H},
$$

where $\alpha=1,2, \nu_{1}+\nu_{2}=1,0 \leq \nu_{i} \leq 1, i=1,2$ and $a_{1}, a_{2}$ are positive constants. Then

$$
\sum_{i=1}^{2}\left[a_{i} \mu_{i} \int_{\substack{i \\ \Omega_{t}}} \stackrel{i}{H}, t \cdot \stackrel{i}{H} d x+a_{i} \int_{\substack{i \\ \Omega_{t}}} \stackrel{i}{E} \cdot \operatorname{rot} \stackrel{i}{H} d x\right]=0 .
$$

Proof. We write equations $1.10{ }_{1,2}$ in the form

$$
\begin{array}{lll}
\mu_{1} \stackrel{1}{H}_{, t}=-\operatorname{rot} \stackrel{1}{E}, & \stackrel{1}{E}=\frac{1}{\sigma_{1}} \operatorname{rot} \stackrel{1}{H}-\mu_{1} v \times \stackrel{1}{H}, \quad \operatorname{div} \stackrel{1}{H}=0 & \text { in } \stackrel{1}{\Omega_{t}} \\
\mu_{2} H_{, t}=-\operatorname{rot} \stackrel{2}{E}, & \stackrel{2}{E}=\frac{1}{\sigma_{2}} \operatorname{rot} \stackrel{2}{H}, \quad \operatorname{div} \stackrel{2}{H}=0 & \text { in } \stackrel{2}{\Omega_{t}} .
\end{array}
$$

Hence

$$
\sum_{i=1}^{2} \int_{\substack{i \\ \Omega_{t}}} a_{i} \mu_{i} \stackrel{i}{H}_{, t} \cdot \stackrel{i}{H} d x+\sum_{i=1}^{2} \int_{\substack{i \\ \Omega_{t}}} a_{i} \operatorname{rot} \stackrel{i}{E} \cdot \stackrel{i}{H} d x=0 .
$$

To obtain an energy type estimate we have to integrate by parts in the second term. Moreover, no boundary term on $S_{t}$ can appear. For this we shall need the transmission condition 2.8. 
Let us recall the identity

$$
\int_{\Omega_{t}} \operatorname{rot} H \cdot \psi d x=\int_{\Omega_{t}} H \cdot \operatorname{rot} \psi d x-\int_{S_{t}} \bar{n} \times H \cdot \psi d S_{t},
$$

where $\bar{n}$ is the unit outward vector to $\Omega_{t}$, normal to $S_{t}$. From 2.13) we have

$$
\begin{aligned}
& \int_{\substack{1 \\
\Omega_{t}}} \operatorname{rot} \stackrel{1}{E} \cdot \stackrel{1}{H} d x=\int_{\substack{1 \\
\Omega_{t}}} \stackrel{1}{E} \cdot \operatorname{rot} \stackrel{1}{H} d x-\int_{S_{t}} \frac{1}{n} \times \stackrel{1}{E} \cdot \stackrel{1}{H} d S_{t}, \\
& \int_{\substack{2 \\
\Omega_{t}}} \operatorname{rot} \stackrel{2}{E} \cdot \stackrel{2}{H} d x=\int_{\substack{2 \\
\Omega_{t}}} \stackrel{2}{E} \cdot \operatorname{rot} \stackrel{2}{H} d x-\int_{S_{t}} \stackrel{2}{n} \times \stackrel{2}{E} \cdot \stackrel{2}{H} d S_{t},
\end{aligned}
$$

where $\frac{i}{n}$ is outward to $\stackrel{i}{\Omega}_{t}$ and $\frac{1}{n}=-\frac{2}{\bar{n}}$. Inserting 2.13) and 2.14 in 2.12 we derive

$$
\begin{aligned}
\sum_{i=1}^{2} \int_{\substack{i \\
\Omega_{t}}} a_{i} \mu_{i} \stackrel{i}{H}, t \cdot \stackrel{i}{H} d x & +\sum_{i=1}^{2} \int_{\substack{i \\
\Omega_{t}}} a_{i} \stackrel{i}{E} \cdot \operatorname{rot} \stackrel{i}{H} d x \\
& -\int_{S_{t}}\left(a_{1} \frac{1}{\bar{n}} \times \stackrel{1}{E} \cdot \stackrel{1}{H}-a_{2} \frac{1}{\bar{n}} \times \stackrel{2}{E} \cdot \stackrel{2}{H}\right) d S_{t}=0 .
\end{aligned}
$$

The boundary term must vanish because otherwise (2.16) does not imply any estimate. The boundary term contains only the tangent components of $\stackrel{i}{E}$ and $\stackrel{i}{H}, i=1,2$. Let $\bar{\tau}_{1}, \bar{\tau}_{2}, \bar{n}$ be an orthonormal system of vectors. Then we have the expansion

$$
\stackrel{i}{E}=\sum_{\alpha=1}^{2} \stackrel{i}{E} \cdot \bar{\tau}_{\alpha} \bar{\tau}_{\alpha}+\stackrel{i}{E} \cdot \bar{n} \bar{n}, \quad i=1,2,
$$

where $\bar{n}=\bar{n}$. Then the boundary term in 2.16 equals

$$
I=-\sum_{\alpha=1}^{2} \int_{S_{t}}\left[a_{1} \stackrel{1}{E} \cdot \bar{\tau}_{\alpha} \bar{n} \times \bar{\tau}_{\alpha} \cdot \stackrel{1}{\bar{H}}-a_{2} \stackrel{2}{E} \cdot \bar{\tau}_{\alpha} \bar{n} \times \bar{\tau}_{\alpha} \cdot \stackrel{2}{H}\right] d S_{t} .
$$

Hence, the transmission conditions (2.8) imply that $I$ vanishes.

In the case $a_{1}=a_{2}=1$, the transmission conditions (2.8) assume the form 1.106 . This concludes the proof.

Let us consider the problem

$$
\begin{array}{ll}
v_{t}-\operatorname{div} \mathbb{T}(v, q)=f & \text { in } \Omega^{T}, \\
\operatorname{div} v=0 & \text { in } \Omega^{T}, \\
\bar{n} \cdot \mathbb{T}(v, q)=g & \text { on } S^{T}, \\
\left.v\right|_{t=0}=v_{0} & \text { in } \Omega .
\end{array}
$$


LeMma 2.5 (see $22 \mid$ ). Assume that $f \in L_{p}\left(\Omega^{T}\right), g \in W_{p}^{1-1 / p, 1 / 2-1 / 2 p}\left(S^{T}\right)$, $v_{0} \in W_{p}^{2-2 / p}(\Omega), p \in(1, \infty), S \in C^{2}$. Then there exists a solution to problem 2.18 such that $v \in W_{p}^{2,1}\left(\Omega^{T}\right), \nabla q \in L_{p}\left(\Omega^{T}\right)$, and

$$
\begin{aligned}
& \|v\|_{W_{p}^{2,1}\left(\Omega^{T}\right)}+\|\nabla q\|_{L_{p}\left(\Omega^{T}\right)} \\
& \quad \leq c\left(\|f\|_{L_{p}\left(\Omega^{T}\right)}+\|g\|_{W_{p}^{1-1 / p, 1 / 2-1 / 2 p}\left(S^{T}\right)}+\left\|v_{0}\right\|_{W_{p}^{2-2 / p}(\Omega)}\right) .
\end{aligned}
$$

3. Method of successive approximations. Let $v_{n}=v_{n}(x, t)$ be given, and $x \in \stackrel{1}{\Omega_{t}}$.

DEFINITION 3.1. Recall that the Lagrangian coordinates in $\stackrel{1}{\Omega}_{0}$ are initial data to the Cauchy problem

$$
\frac{d x}{d t}=v_{n}(x, t),\left.\quad x\right|_{t=0}=\xi \in \stackrel{1}{\Omega_{0}} .
$$

Hence

$$
\stackrel{1}{\Omega}_{n t}=\left\{x \in \mathbb{R}^{3}: x=x^{(n)}(\xi, t)=\xi+\int_{0}^{t} \bar{v}_{n}\left(\xi, t^{\prime}\right) d t^{\prime}, \xi \in \stackrel{1}{\Omega}_{0}\right\},
$$

where $\bar{v}_{n}(\xi, t)=v_{n}\left(x^{(n)}(\xi, t), t\right)$.

In free boundary problems in hydrodynamics the free boundary is built up from the same fluid particles because $\left.v_{n}\right|_{S_{n t}}$ is tangent to $S_{n t}$ and

$$
S_{n t}=\left\{x \in \mathbb{R}^{3}: x=x^{(n)}(\xi, t), \xi \in S_{0}\right\} .
$$

To formulate problem 1.10 in Lagrangian coordinates we have to introduce them in $\stackrel{2}{\Omega_{0}}$. Since there is no velocity in $\stackrel{2}{\Omega}_{t}$, we have to introduce it artificially (see Lemma 2.1).

Definition 3.2. Let us denote $\stackrel{1}{v}_{n}=v_{n}$ in $\stackrel{1}{\Omega_{t}}$ and define $\stackrel{2}{v}_{n}$ in $\stackrel{2}{\Omega}_{t}$ as a solution to the nonstationary Stokes system

$$
\begin{array}{ll}
\stackrel{2}{v}_{n, t}-\operatorname{div} \mathbb{T}\left(\hat{v}_{n}, q_{n}\right)=0 & \text { in } \stackrel{2}{\Omega_{t},}, \\
\operatorname{div} \stackrel{2}{v}_{n}=0 & \text { in } \stackrel{2}{\Omega_{t},}, \\
\left.\stackrel{2}{v}_{n}\right|_{S_{t}}=\left.{ }_{v}\right|_{S_{t}},\left.\quad \stackrel{2}{v}_{n}\right|_{B}=0, & \\
\left.\stackrel{2}{v}_{n}\right|_{t=0}=\stackrel{2}{v}(0) & \text { in } \stackrel{2}{\Omega_{0},},
\end{array}
$$

where $q_{n}$ plays the role of pressure but it is not important for any estimate for $\stackrel{2}{v}_{n}$. The initial data $\stackrel{2}{v}(0)$ is an extension of $\stackrel{1}{v}(0)$ through the given fixed boundary $S_{0}$, because $\left.\stackrel{2}{v}(0)\right|_{S_{0}}=\left.\stackrel{1}{v}(0)\right|_{S_{0}}$. The extension can be made by applying Lemma 2.1. The existence of solutions to 3.2 follows from Lemma 2.3 . 
Now, we introduce the Lagrangian coordinates $\stackrel{1}{\xi}, \stackrel{2}{\xi}$ as the initial data to the problems

$$
\frac{d x}{d t}=\stackrel{i}{v}_{n}(x, t),\left.\quad x^{i}\right|_{t=0}=\stackrel{i}{\xi} \in \stackrel{i}{\Omega_{0}}, \quad i=1,2 .
$$

Then

$$
\begin{aligned}
\stackrel{i}{\Omega_{n t}}=\left\{\stackrel{i}{x} \in \mathbb{R}^{3}: \stackrel{i}{x}=\stackrel{i}{x}(n) \stackrel{i}{\xi}, t\right)=\stackrel{i}{\xi} & +\int_{0}^{t} \stackrel{i}{v}_{n}\left(\stackrel{i}{x}, t^{\prime}\right) d t^{\prime} \\
& \left.=\stackrel{i}{\xi}+\int_{0}^{t} \frac{i}{v_{n}}\left(\xi, t^{\prime}\right) d t^{\prime}, \stackrel{i}{\xi} \in \stackrel{i}{\Omega_{0}}\right\},
\end{aligned}
$$

where $\left.\stackrel{i}{v_{n}}(\stackrel{i}{\xi}, t)=\stackrel{i}{v}_{n}(\stackrel{i}{x}(n) \stackrel{i}{\xi}, t), t\right), \stackrel{i}{\xi} \in \stackrel{i}{\Omega^{\prime}}, i=1,2$.

Formulation of the method of successive approximations. Let $v$ in problem 1.10 be given. We set $v=v_{n}$. To emphasize that $v_{n}$ describes the motion in $\stackrel{1}{\Omega}_{t}$ we write $v_{n}=\stackrel{1}{v}_{n}$. Then by Definition 3.2 we have $\stackrel{2}{v}_{n}$ in $\stackrel{2}{\Omega}_{t}$. Passing to the Lagrangian coordinates expressed by (3.3), (3.4) we can write problem 1.10 as a problem for $\stackrel{i}{H}_{n}, i=1,2$, in the form

$$
\begin{aligned}
& \mu_{1} \stackrel{1}{\bar{H}}_{n t}+\operatorname{rot}_{\bar{v}_{n}}\left[\frac{1}{\sigma_{1}}\left(\operatorname{rot}_{\bar{v}_{n}} \stackrel{1}{\bar{H}}_{n}-\mu_{1} \stackrel{1}{\bar{v}}_{n} \times \stackrel{1}{\bar{H}}_{n}\right)\right] \\
& =\mu_{1} \frac{1}{v}_{n} \cdot \nabla_{\frac{1}{v}_{n}} \stackrel{1}{\bar{H}}_{n}, \quad \operatorname{div}_{\frac{1}{\bar{v}_{n}}} \stackrel{1}{\bar{H}}_{n}=0 \quad \text { in } \stackrel{1}{\Omega}_{0} \times(0, t), \\
& \mu_{2} \stackrel{2}{H}_{n t}+\operatorname{rot}_{\bar{v}_{n}}\left[\frac{1}{\sigma_{2}} \operatorname{rot}_{\bar{v}_{n}} \stackrel{2}{\bar{H}}\right] \\
& \begin{array}{c}
=\mu_{2} \bar{v}_{n} \cdot \nabla_{\bar{v}_{n}} \stackrel{2}{\bar{H}}_{n}, \operatorname{div}_{\bar{v}_{n}} \stackrel{2}{\bar{H}}_{n}=0 \\
\left(\operatorname{rot}_{\bar{v}_{n}} \stackrel{1}{\bar{H}}_{n}-\mu_{1}{ }^{\frac{1}{v_{n}}} \times \stackrel{1}{\bar{H}}_{n}\right) \cdot \bar{\tau}_{\bar{v}_{n} \alpha}
\end{array} \\
& =\frac{1}{\sigma_{2}} \operatorname{rot}_{\bar{v}_{n}} \stackrel{2}{\bar{H}_{n}} \cdot \bar{\tau}_{\bar{v}_{n} \alpha}, \quad \alpha=1,2, \quad \text { on } S_{0} \times(0, t), \\
& \bar{n}_{\bar{v}_{n}} \times \bar{\tau}_{\bar{v}_{n} \alpha} \cdot(\stackrel{1}{\bar{H}}-\stackrel{2}{\bar{H}})=0, \quad \alpha=1,2, \quad \text { on } S_{0} \times(0, t), \\
& \mu_{1} \bar{n}_{\bar{v}_{n}} \cdot \stackrel{1}{\bar{H}}_{n}=\mu_{2} \bar{n}_{\bar{v}_{n}} \cdot \stackrel{2}{\bar{H}}_{n} \quad \text { on } S_{0} \times(0, t), \\
& \left.\stackrel{2}{\bar{H}}_{n}\right|_{B}=0 \\
& \text { on } B \times(0, t) \text {, } \\
& \left.\stackrel{i}{H}\right|_{t=0}=\stackrel{i}{H}(0), \\
& \text { in } \stackrel{i}{\Omega_{0}}, i=1,2 \text {, }
\end{aligned}
$$


where $\nabla_{\bar{v}_{n}}=\left.\frac{\partial \xi}{\partial x^{(n)}}\right|_{x^{(n)}=x^{(n)}(\xi, t)} \cdot \nabla_{\xi}$ and any operator with index $\bar{v}_{n}$ means that it contains the transformed gradient $\nabla_{\bar{v}_{n}}$. Moreover, $\bar{v}_{n}=\frac{2}{v}_{n}=\bar{v}_{n}$ on $S_{0}$.

For $\bar{v}_{n}$ given we have $\stackrel{i}{v}_{n}, i=1,2$, and also the domains $\stackrel{i}{\Omega}_{n t}, i=1,2$, defined by 3.4. Hence, in Lagrangian coordinates, problem 3.5 for the quantities $\stackrel{i}{H}_{n}, i=1,2$, is formulated in the fixed (independent of time) domains $\stackrel{i}{\Omega_{0}}, i=1,2$.

We have to emphasize that the terms on the r.h.s. of $(3.5)_{1,2}$ follow from expressing problem (1.10 in Lagrangian coordinates. This means that the Lagrangian coordinates for problem $(1.10)$ are introduced artificially. But this is done because formulation of a free boundary problem for the fluid mechanics equations in these coordinates is very natural and simple. The main point is that the problem derived is formulated in a fixed initial domain.

For given $\bar{v}_{n}, i=1,2$, problem 3.5 for variable $\bar{H}_{n}^{i}, i=1,2$, is linear. However, to prove existence of solutions we have to use the method of successive approximations. For this purpose we write problem 3.5 in the form

$$
\begin{aligned}
& \mu_{1} \stackrel{1}{\bar{H}}_{n t}+\frac{1}{\sigma_{1}} \operatorname{rot}_{\xi}^{2} \stackrel{1}{\bar{H}}_{n}=\frac{1}{\sigma_{1}}\left(\operatorname{rot}_{\xi}^{2} \stackrel{1}{\bar{H}}_{n}-\operatorname{rot}_{\underline{\bar{v}}_{n}}^{2} \stackrel{1}{\bar{H}}_{n}\right) \\
& +\mu_{1} \operatorname{rot}_{\bar{v}_{n}}\left(\bar{v}_{n} \times \stackrel{1}{\bar{H}}_{n}\right)+\mu_{1} \bar{v}_{n} \cdot \nabla_{\frac{1}{v}_{n}} \stackrel{1}{\bar{H}}_{n} \equiv \stackrel{1}{f}, \\
& \operatorname{div}_{\xi} \stackrel{1}{\bar{H}}_{n}=\operatorname{div}_{\xi} \stackrel{1}{\bar{H}}_{n}-\operatorname{div}_{\bar{v}_{n}} \stackrel{1}{\bar{H}}_{n} \equiv \stackrel{1}{g} \quad \text { in } \stackrel{1}{\Omega}_{0} \times(0, t), \\
& \mu_{2} \stackrel{2}{\bar{H}}_{n t}+\frac{1}{\sigma_{2}} \operatorname{rot}_{\xi}^{2} \stackrel{2}{\bar{H}}_{n}=\frac{1}{\sigma_{2}}\left(\operatorname{rot}_{\xi}^{2} \stackrel{2}{\bar{H}}_{n}-\operatorname{rot}_{\underline{v}_{n}}^{2} \stackrel{2}{\bar{H}}_{n}\right) \\
& +\mu_{2} \stackrel{2}{\bar{v}}_{n} \cdot \nabla_{\bar{v}_{n}} \stackrel{2}{\bar{H}}_{n} \equiv \stackrel{2}{f} \\
& \operatorname{div}_{\xi} \stackrel{2}{\bar{H}_{n}}=\operatorname{div}_{\xi} \stackrel{2}{\bar{H}}_{n}-\operatorname{div}_{\bar{v}_{n}} \stackrel{2}{\bar{H}_{n}} \equiv \stackrel{2}{g} \quad \text { in } \stackrel{2}{\Omega_{0}} \times(0, t), \\
& \left(\frac{1}{\sigma_{1}} \operatorname{rot}_{\xi} \stackrel{1}{\bar{H}}_{n}-\frac{1}{\sigma_{2}} \operatorname{rot}_{\xi} \stackrel{2}{\bar{H}}_{n}\right) \cdot \bar{\tau}_{\alpha} \\
& =\frac{1}{\sigma_{1}}\left(\operatorname{rot}_{\xi} \frac{1}{\bar{H}_{n}} \cdot \bar{\tau}_{\alpha}-\operatorname{rot}_{\bar{v}_{n}} \stackrel{1}{H}_{n} \cdot \bar{\tau}_{\bar{v}_{n} \alpha}\right) \\
& -\frac{1}{\sigma_{2}}\left(\operatorname{rot}_{\xi} \stackrel{2}{\bar{H}_{n}} \cdot \bar{\tau}_{\alpha}-\operatorname{rot}_{\bar{v}_{n}} \stackrel{2}{H}_{n} \cdot \bar{\tau}_{\bar{v}_{n} \alpha}\right) \\
& +\frac{\mu_{1}}{\sigma_{1}} \bar{v}_{n} \times \stackrel{1}{\bar{H}}_{n} \cdot \bar{\tau}_{\bar{v}_{n} \alpha} \equiv g_{\alpha}, \quad \alpha=1,2, \quad \text { on } S_{0} \times(0, t),
\end{aligned}
$$


[cont.]

$$
\begin{aligned}
& \bar{n} \times \bar{\tau}_{\alpha} \cdot\left(\overline{\bar{H}}_{n}-\stackrel{2}{\bar{H}}_{n}\right) \\
& =\left(\bar{n} \times \bar{\tau}_{\alpha}-\bar{n}_{\bar{v}_{n}} \times \bar{\tau}_{\bar{v}_{n} \alpha}\right) \cdot\left(\frac{1}{\bar{H}_{n}}-\stackrel{2}{\bar{H}}_{n}\right) \equiv k_{\alpha}, \quad \alpha=1,2, \quad \text { on } S_{0} \times(0, t), \\
& \mu_{1} \bar{n} \cdot \stackrel{1}{H}_{n}-\mu_{2} \bar{n} \cdot \stackrel{2}{\bar{H}}_{n} \\
& =\mu_{1}\left(\bar{n}-\bar{n}_{\bar{v}_{n}}\right) \cdot \stackrel{1}{\bar{H}}_{n}-\mu_{2}\left(\bar{n}-\bar{n}_{\bar{v}_{n}}\right) \cdot \stackrel{2}{\bar{H}} \equiv l \quad \text { on } S_{0} \times(0, t), \\
& \left.\stackrel{2}{\bar{H}}_{n}\right|_{B}=0 \\
& \text { on } B \times(0, t) \text {, } \\
& \left.\stackrel{i}{H}_{n}\right|_{t=0}=\stackrel{i}{H}(0) \\
& \text { in } \stackrel{i}{\Omega}_{0}, i=1,2 \text {. }
\end{aligned}
$$

For given $\frac{1}{v_{n}}, \frac{2}{v}_{n}$ we prove existence of solutions to problem 3.6 in two steps. First we consider the problem with constant coefficients

$$
\begin{aligned}
& \mu_{i} \stackrel{i}{H}_{n t}+\frac{1}{\sigma_{i}} \operatorname{rot}_{\xi}^{2} \stackrel{i}{\bar{H}}_{n}=\stackrel{i}{f}, \quad \operatorname{div}_{\xi} \stackrel{i}{\bar{H}}_{n}=\stackrel{i}{g} \quad \text { in } \stackrel{i}{\Omega}_{0} \times(0, t), i=1,2, \\
& \left(\frac{1}{\sigma_{1}} \operatorname{rot}_{\xi} \stackrel{1}{\bar{H}}_{n}-\frac{1}{\sigma_{2}} \operatorname{rot}_{\xi} \stackrel{2}{\stackrel{H}{H}_{n}}\right) \cdot \bar{\tau}_{\alpha}=g_{\alpha}, \quad \alpha=1,2, \quad \text { on } S_{0} \times(0, t), \\
& \left(\bar{n} \times \bar{\tau}_{\alpha}\right) \cdot\left(\stackrel{1}{\bar{H}}_{n}-\stackrel{2}{\bar{H}}_{n}\right)=k_{\alpha}, \quad \alpha=1,2, \quad \text { on } S_{0} \times(0, t), \\
& \mu_{1} \bar{n} \cdot \stackrel{1}{H}_{n}=\mu_{2} \bar{n} \cdot \stackrel{2}{H}_{n} \quad \text { on } S_{0} \times(0, t), \\
& \stackrel{2}{\bar{H}}_{n}=0 \\
& \left.\stackrel{i}{H}_{n}\right|_{t=0}=\stackrel{i}{H}(0) \\
& \text { on } B \times(0, t) \text {, } \\
& \text { in } \stackrel{i}{\Omega_{0}}, i=1,2 \text {. }
\end{aligned}
$$

Existence of solutions to (3.7) can be shown either by applying the FaedoGalerkin method (see [5]) or by the technique of regularizer (see [20, 21]). To use the Faedo-Galerkin method we need a fundamental basis for problem (3.7). Existence of such a basis will be shown in Section 5 .

Having existence of solutions to problem (3.7) with appropriately regular r.h.s. functions we can show existence of solutions to problem (3.6) by the method of successive approximations for sufficiently small time. For this we replace in the r.h.s. functions of $3.6 \frac{i}{\bar{H}_{n}}$ by $\stackrel{i}{\bar{H}}_{n}^{(m)}, m \in \mathbb{N}$, and in the l.h.s. functions by $\stackrel{i}{H}_{n}^{(m+1)}$. Then problem 3.6 leads to a mapping

$$
\left(\overline{\bar{H}}_{n}^{(m+1)}, \stackrel{2}{\bar{H}}_{n}^{(m+1)}\right)=\Phi\left(\overline{\bar{H}}_{n}^{(m)}, \stackrel{2}{\bar{H}}_{n}^{(m)}\right),
$$

which for sufficiently small time and regular $\frac{1}{\bar{v}_{n}}, \frac{2}{v}_{n}$ has a fixed point given by 
the method of successive approximations. In this way existence of solutions to problem (3.6) is proved and the following functional dependence holds:

$$
\left(\stackrel{1}{H}_{n}, \stackrel{2}{\bar{H}}_{n}\right)=F\left(\bar{v}_{n}\right)
$$

where we have used the facts that $\frac{1}{v}_{n}=\bar{v}_{n}$ and $\frac{2}{v}_{n}$ is described by problem 3.2 .

The functional dependence $(3.9)$ is expressed by the estimate

$$
\sum_{i=1}^{2}\left\|\stackrel{i}{H}_{n}\right\|_{1, \Omega_{0}^{t}} \leq \varphi\left(\left\|\bar{v}_{n}\right\|_{2, \Omega_{0}^{t}}\right),
$$

where the norms \|\|$_{1, \Omega_{0}^{t}},\|\|_{2, \Omega_{0}^{t}}, \stackrel{i}{\Omega_{0}^{t}}=\stackrel{i}{\Omega_{0}} \times(0, t), i=1,2$, are found in [5, 20, 21] and $\varphi$ is some increasing positive function.

To calculate the next approximation $v_{n+1}$ in the method of successive approximations applied to problem (1.1)-(1.8), we use problem (1.9) . Expressing (1.9) in Lagrangian coordinates we have

$$
\begin{array}{ll}
\bar{v}_{n+1, t}-\operatorname{div}_{\bar{v}_{n}} \mathbb{T}_{\bar{v}_{n}}\left(\bar{v}_{n+1}, \bar{p}_{n+1}\right)=\bar{f}+\mu_{1} \operatorname{div}_{\bar{v}_{n}} \mathbb{T}\left(\overline{\bar{H}}_{n}\right) & \text { in } \stackrel{1}{\Omega}_{0}^{t}, \\
\operatorname{div}_{\bar{v}_{n}} \bar{v}_{n+1}=0 & \text { in } \Omega_{0}^{t}, \\
\bar{n}_{\bar{v}_{n}} \cdot \mathbb{T}_{\bar{v}_{n}}\left(\bar{v}_{n+1}, \bar{p}_{n+1}\right)=p_{0} \bar{n}_{\bar{v}_{n}}-\mu_{1} \bar{n}_{\bar{v}_{n}} \mathbb{T}\left(\bar{H}_{n}\right) & \text { on } S_{0}^{t}, \\
\left.\bar{v}_{n+1}\right|_{t=0}=v(0) & \text { in } \stackrel{1}{\Omega}_{0},
\end{array}
$$

where we have used the simplified notation $\bar{v}_{n}=\frac{1}{v}_{n}, \bar{v}_{n+1}=\frac{1}{n+1}_{n+1}$. Moreover, $\bar{v}_{n}$ and $\stackrel{1}{\bar{H}}_{n}$ are given, where $\bar{v}_{n}$ denotes the $n$th successive approximation and $\stackrel{1}{\bar{H}}$ depends on $\bar{v}_{n}$ by formula 3.9 .

To prove existence of solutions to problem (3.11) we write it in the form

$$
\begin{array}{cc}
\bar{v}_{n+1, t}-\operatorname{div}_{\xi} \mathbb{T}_{\xi}\left(\bar{v}_{n+1}, \bar{p}_{n+1}\right)=-\left(\operatorname{div}_{\xi} \mathbb{T}_{\xi}\left(\bar{v}_{n+1}, \bar{p}_{n+1}\right)\right. & \\
\left.-\operatorname{div}_{\bar{v}_{n}} \mathbb{T}_{\bar{v}_{n}}\left(\bar{v}_{n+1}, \bar{p}_{n+1}\right)\right)+\mu_{1} \operatorname{div}_{\bar{v}_{n}} \mathbb{T}\left(\overline{\bar{H}}_{n}\right)+\bar{f} \equiv f_{0} & \text { in } \Omega_{0}^{t}, \\
\operatorname{div}_{\xi} \bar{v}_{n+1}=\operatorname{div}_{\xi} \bar{v}_{n+1}-\operatorname{div}_{\bar{v}_{n}} \bar{v}_{n+1} \equiv g_{0} & \text { in } \stackrel{1}{\Omega}_{0}^{t}, \\
\bar{n}_{\xi} \mathbb{T}_{\xi}\left(\bar{v}_{n+1}, \bar{p}_{n+1}\right)=\bar{n}_{\xi} \mathbb{T}_{\xi}\left(\bar{v}_{n+1}, \bar{p}_{n+1}\right)-\bar{n}_{\bar{v}_{n}} \mathbb{T}_{\bar{v}_{n}}\left(\bar{v}_{n+1}, \bar{p}_{n+1}\right) & \\
+p_{0} \bar{n}_{\bar{v}_{n}}-\mu_{1} \bar{n}_{\bar{v}_{n}} \cdot \mathbb{T}\left(\bar{H}_{n}\right) \equiv k_{0} & \text { on } S_{0}^{t}, \\
\left.\bar{v}_{n+1}\right|_{t=0}=v(0) & \text { in } \stackrel{1}{\Omega_{0} .} .
\end{array}
$$


To prove existence of solutions to problem 3.12 we repeat the approach applied to problem (3.6). Thus, we first consider the problem

$$
\begin{aligned}
& \bar{v}_{n+1, t}-\operatorname{div}_{\xi} \mathbb{T}_{\xi}\left(\bar{v}_{n+1}, \bar{p}_{n+1}\right)=f_{0}, \\
& \operatorname{div}_{\xi} \bar{v}_{n+1}=g_{0}, \\
& \bar{n}_{\xi} \cdot \mathbb{T}_{\xi}\left(\bar{v}_{n+1}, \bar{p}_{n+1}\right)=k_{0}, \\
& \left.\bar{v}_{n+1}\right|_{t=0}=v(0) .
\end{aligned}
$$

Existence of solutions to problem $(3.13)$ can be proved either by the FaedoGalerkin method (see Section 4 ) or by the technique of regularizer (see Lemma 2.5). Having existence of solutions to problem (3.13) we can prove existence of solutions to (3.12) by the method of successive approximations such that the r.h.s. functions depend on $\bar{v}_{n+1}^{(m)}, \bar{p}_{n+1}^{(m)}$ and the l.h.s. on $\bar{v}_{n+1}^{(m+1)}$, $\bar{p}_{n+1}^{(m+1)}$. Hence 3.12 leads to a mapping

$$
\left(\bar{v}_{n+1}^{(m+1)}, \bar{p}_{n+1}^{(m+1)}\right)=\Phi\left(\bar{v}_{n+1}^{(m)}, \bar{p}_{n+1}^{(m)}\right),
$$

where $m \in \mathbb{N}$. Hence for sufficiently small time and given $\bar{v}_{n}$ the mapping (3.14) has a fixed point which is a solution to problem (3.12) for given $\bar{v}_{n}$. Then we get the functional dependence

$$
\bar{v}_{n+1}=F\left(\bar{v}_{n}\right) .
$$

Hence, again, by the method of successive approximations and the assumption that $\bar{v}_{0}$ is some extension of the initial data we show for sufficiently small time the existence of a fixed point of the mapping (3.15). In this way we show existence of solutions to problem (1.1)-(1.8).

4. Existence of solutions to problem (3.13). For simplicity we write problem 3.13) in the form

$$
\begin{aligned}
& v_{t}-\operatorname{div}_{\xi} \mathbb{T}_{\xi}(v, p)=f_{0}, \\
& \operatorname{div}_{\xi} v=g_{0}, \\
& \bar{n}_{\xi} \cdot \mathbb{T}_{\xi}(v, p)=k_{0}, \\
& \left.v\right|_{t=0}=v(0)
\end{aligned}
$$

We construct a function $G$ satisfying the problem

$$
\begin{aligned}
& \operatorname{div}_{\xi} G=g_{0}, \\
& \left.G\right|_{S_{0}}=0 .
\end{aligned}
$$

By applying the Bogovskiǔ operator $B$ (see also [11]), solutions to 4.2 can be written in the form

$$
G=B * g_{0}
$$

Introducing the new function

$$
u=v-G
$$


we see that $(u, p)$ is a solution to the problem

$$
\begin{aligned}
& u_{, t}-\operatorname{div}_{\xi} \mathbb{T}_{\xi}(u, p)=-G_{, t}+\operatorname{div}_{\xi} \mathbb{D}(G)+f_{0} \equiv F, \\
& \operatorname{div} u=0, \\
& \bar{n}_{\xi} \cdot \mathbb{T}_{\xi}(u, p)=-\bar{n}_{\xi} \mathbb{D}_{\xi}(G)+k_{0} \equiv H, \\
& \left.u\right|_{t=0}=v(0)-\left.G\right|_{t=0} \equiv u(0) .
\end{aligned}
$$

Simplifying (4.5) yields

$$
\begin{array}{ll}
u_{, t}-\operatorname{div}_{\xi} \mathbb{T}_{\xi}(u, p)=F & \text { in } \stackrel{1}{\Omega}_{0}^{T}, \\
\operatorname{div} u=0 & \text { in } \stackrel{1}{\Omega}_{0}^{T}, \\
\bar{n}_{\xi} \cdot \mathbb{T}_{\xi}(u, p)=H & \text { on } S_{0}^{T}, \\
\left.u\right|_{t=0}=u(0) & \text { in } \stackrel{1}{\Omega}_{0} .
\end{array}
$$

For the sake of [5] we solve the problem using the Faedo-Galerkin method. The Faedo-Galerkin method implies existence of solutions to problem 4.6 via the $L_{2}$-approach.

Definition 4.1. By a weak solution to problem (4.6) we mean any solution to the integral identity

$$
\int_{\substack{1 \\ \Omega_{0}^{T}}} u, t \cdot \eta d x d t+\int_{\Omega_{0}^{T}} \mathbb{D}(u) \cdot \mathbb{D}(\eta) d x d t=\int_{S_{0}^{T}} H \cdot \eta d S_{0} d t+\int_{\substack{1 \\ \Omega_{0}^{T}}} F \cdot \eta d x d t
$$

where $B^{T}=B \times[0, T]$, which holds for any $\eta \in L_{2}\left(0, T ; H^{1}\left(\stackrel{1}{\Omega_{0}}\right) \cap V\left(\stackrel{1}{\Omega_{0}}\right)\right)$, where the time derivative is understood in the weak sense and the following spaces are introduced:

$$
\vartheta=\left\{u \in C^{\infty}\left(\stackrel{1}{\Omega_{0}}\right): \operatorname{div} u=0\right\}, \quad V=\text { closure of } \vartheta \text { in } W_{2}^{1}\left(\stackrel{1}{\Omega_{0}}\right) .
$$

Since $V$ is separable, there exists a sequence of linearly independent elements $\varphi_{1}, \ldots, \varphi_{m}, \ldots$, which is a basis in $V$. The existence of a fundamental basis for the Stokes system (4.6) is proved in 25].

Therefore, we are looking for approximate solutions to 4.7 in the form

$$
\begin{aligned}
& u_{m}=\sum_{i=1}^{m} c_{i m}(t) \varphi_{i}(x) \\
& \sum_{i=1}^{m} \dot{c}_{i m} \int_{\substack{1 \\
\Omega_{0}}} \varphi_{i} \cdot \varphi_{l} d x+\sum_{i=1}^{m} c_{i m} \int_{\substack{1 \\
\Omega_{0}}} \mathbb{D}\left(\varphi_{i}\right) \cdot \mathbb{D}\left(\varphi_{l}\right) d x \\
& =\int_{S_{0}} H \cdot \varphi_{l} d S_{0}+\int_{\substack{1 \\
\Omega_{0}}} F \cdot \varphi_{l} d x, \quad l=1, \ldots, m,\left.\quad u_{m}\right|_{t=0}=u_{m}(0)
\end{aligned}
$$

and $\dot{c}_{i m}=c_{i m, t}$. 
Since $\varphi_{1}, \ldots, \varphi_{m}$ are linearly independent, we have $\operatorname{det} \int_{\Omega} \varphi_{i} \cdot \varphi_{j} d x \neq 0$. Therefore, (4.8) yields the following linear system with constant coefficients and time dependent r.h.s.:

$$
\begin{aligned}
& \dot{c}_{i m}+\sum_{j=1}^{m} \alpha_{i j} c_{j m}=\sum_{j=1}^{m} \beta_{i j} K_{j}, \quad i=1, \ldots, m, \\
& \left.c_{i m}\right|_{t=0}=c_{i m}(0),
\end{aligned}
$$

where

$$
K_{j}=\int_{S_{0}} H \cdot \varphi_{j} d S+\int_{\substack{1 \\ \Omega_{0}}} F \cdot \varphi_{j} d x, \quad u_{m}(0)=\sum_{i=1}^{m} c_{i m}(0) \varphi_{i}
$$

Lemma 4.2. Assume that $H \in L_{2}\left(0, T ; L_{2}\left(S_{0}\right)\right), F \in L_{2}\left(0, T ; L_{2}\left(\stackrel{1}{\Omega_{0}}\right)\right)$, $u(0) \in L_{2}\left(\stackrel{1}{\Omega}{ }_{0}\right)$. Then there exists a weak solution to problem 4.6 such that $u \in V_{2}^{0}\left(\stackrel{1}{\Omega}{ }_{0}^{T}\right)$, and we have the estimate

$$
\|u\|_{V_{2}^{0}\left(\Omega_{0}^{T}\right)} \leq c\left(\|F\|_{L_{2}\left(\Omega_{0}^{T}\right)}+\|H\|_{L_{2}\left(S_{0}^{T}\right)}+\|u(0)\|_{L_{2}\left(\Omega_{0}\right)}\right) .
$$

Proof. Since 4.9 is a system of linear ordinary differential equations, the existence of solutions is well known. To prove existence of weak solutions in $V_{2}^{0}\left(\stackrel{1}{\Omega}_{0}^{T}\right)$ we multiply 4.8 by $c_{l m}$ and sum over $l$ from 1 to $m$. Integrating the result with respect to time we get

$$
\left\|u_{m}\right\|_{V_{2}^{0}\left(\Omega_{0}^{T}\right)} \leq c\left(\|F\|_{L_{2}\left(\Omega_{0}^{T}\right)}+\|H\|_{L_{2}\left(S_{0}^{T}\right)}+\|u(0)\|_{L_{2}\left(\Omega_{0}\right)}\right),
$$

where we have used

$$
\left\|u_{m}(0)\right\|_{L_{2}\left(\Omega_{0}\right)} \leq\|u(0)\|_{L_{2}\left(\Omega_{0}\right)} .
$$

Using the weak convergence in $L_{2}\left(0, T ; H^{1}\left(\stackrel{1}{\Omega_{0}}\right)\right)$ and weak star convergence in $L_{\infty}\left(0, T ; L_{2}\left(\stackrel{1}{\Omega_{0}}\right)\right)$ we show that the limit function belongs to $V_{2}^{0}\left(\stackrel{1}{\Omega}_{0}^{T}\right)$ and estimate 4.10 holds. This concludes the proof.

5. Existence of solutions to problem (3.7). Dropping the index $n$ in (3.7) we write it in the simple form

$$
\begin{array}{ll}
\mu_{i} \stackrel{i}{H}_{t}+\frac{1}{\sigma_{i}} \operatorname{rot}_{\xi}^{2} \stackrel{i}{H}=\stackrel{i}{f}, \quad \operatorname{div}_{\xi} \stackrel{i}{H}=\stackrel{i}{g} & \text { in } \stackrel{i}{\Omega_{0}^{t}}, i=1,2, \\
\left(\frac{1}{\sigma_{1}} \operatorname{rot}_{\xi} \stackrel{1}{H}-\frac{1}{\sigma_{2}} \operatorname{rot}_{\xi} \stackrel{2}{H}\right) \cdot \bar{\tau}_{\alpha}=g_{\alpha}, \quad \alpha=1,2, & \text { on } S_{0}^{t}, \\
\bar{n} \times \bar{\tau}_{\alpha} \cdot(\stackrel{1}{H}-\stackrel{2}{H})=k_{\alpha}, \quad \alpha=1,2, & \text { on } S_{0}^{t},
\end{array}
$$


[cont.]

$$
\begin{array}{ll}
\mu_{1} \bar{n} \cdot \stackrel{1}{H}-\mu_{2} \bar{n} \cdot \stackrel{1}{H}=l & \text { on } S_{0}^{t}, \\
\left.\stackrel{2}{H}\right|_{B}=0 & \text { on } B^{t}, \\
\left.\stackrel{i}{H}\right|_{t=0}=\stackrel{i}{H}(0) & \text { in } \stackrel{i}{\Omega_{0}}, i=1,2,
\end{array}
$$

where $\stackrel{i}{H}$ was replaced by $\stackrel{i}{H}, i=1,2$.

To prove existence of solutions to problem (5.1) by the Faedo-Galerkin method we need a weak formulation of problem (5.1). For this purpose we multiply $(5.1)$ by $\stackrel{i}{\psi}$, a sufficiently regular function, and integrate over $\stackrel{i}{\Omega_{0}}$, $i=1,2$. Then

$$
\sum_{i=1}^{2} \int_{\substack{i \\ \Omega_{0}}}\left(\mu_{i} \stackrel{i}{H}_{t}+\frac{1}{\sigma_{i}} \operatorname{rot}_{\xi}^{2} \stackrel{i}{H}\right) \cdot \stackrel{i}{\psi} d \xi=\sum_{i=1}^{2} \int_{\substack{i \\ \Omega_{0}}}^{i} f \cdot \stackrel{i}{f} d \xi
$$

Integration by parts yields

$$
\begin{aligned}
\sum_{i=1}^{2} \int_{\substack{i \\
\Omega_{0}}}\left(\mu_{i} \stackrel{i}{H}_{t} \cdot \stackrel{i}{\psi}\right. & \left.+\frac{1}{\sigma_{i}} \operatorname{rot}_{\xi} \stackrel{i}{H} \cdot \operatorname{rot} \stackrel{i}{\psi}\right) d \xi \\
& -\sum_{i=1}^{2} \int_{S_{0}} \frac{1}{\sigma_{i}} \stackrel{i}{n} \times \operatorname{rot} \stackrel{i}{H} \cdot \stackrel{i}{\psi} d S_{0}=\sum_{i=1}^{2} \int_{\substack{i \\
\Omega_{0}}}^{i} \cdot \stackrel{i}{f} d \xi,
\end{aligned}
$$

where $\frac{i}{n}$ is the unit vector normal to $S_{0}$ outward to $\stackrel{i}{\Omega_{0}}$. Therefore choosing $\bar{n}=\frac{1}{n}$ we get $\frac{2}{n}=-\bar{n}$. Then the boundary term in 5.3 takes the form

$$
I=-\int_{S_{0}} \frac{1}{\sigma_{1}} \bar{n} \times \operatorname{rot} \stackrel{1}{H} \cdot \stackrel{1}{\psi} d \xi+\int_{S_{0}} \frac{1}{\sigma_{2}} \bar{n} \times \operatorname{rot} \stackrel{2}{H} \cdot \stackrel{2}{\psi} d \xi
$$

In the two integrals in $I$ the tangent coordinates of $\stackrel{1}{\psi}$ and $\stackrel{2}{\psi}$ appear only. Therefore, if we use the decomposition

$$
\stackrel{i}{\psi}=\sum_{\alpha=1}^{2} \stackrel{i}{\psi} \cdot \bar{\tau}_{\alpha} \bar{\tau}_{\alpha}+\psi \cdot \bar{n} \bar{n}
$$

the expression $I$ takes the form

$$
I=\sum_{\alpha=1}^{2}\left[-\int_{S_{0}} \frac{1}{\sigma_{1}} \bar{n} \times \operatorname{rot} \stackrel{1}{H} \cdot \bar{\tau}_{\alpha} \stackrel{1}{\psi} \cdot \bar{\tau}_{\alpha} d S_{0}+\int_{S_{0}} \frac{1}{\sigma_{2}} \bar{n} \times \operatorname{rot} \stackrel{2}{H} \cdot \bar{\tau}_{\alpha} \stackrel{2}{\psi} \cdot \bar{\tau}_{\alpha} d S_{0}\right] .
$$

In view of the transmission conditions (1.8) we have

$$
\stackrel{1}{E_{\tau_{\alpha}}}=\stackrel{2}{E}_{\tau_{\alpha}}, \quad \stackrel{1}{H}_{\tau_{\alpha}}=\stackrel{2}{H}_{\tau_{\alpha}}, \quad \alpha=1,2, \quad \text { on } S_{0} .
$$


Recalling that

$$
\stackrel{1}{E}_{\tau_{\alpha}}=\left(\frac{1}{\sigma_{1}} \operatorname{rot} \stackrel{1}{H}-\mu_{1} v \times \stackrel{1}{H}\right) \cdot \bar{\tau}_{\alpha}, \quad \stackrel{2}{E}_{\tau_{\alpha}}=\frac{1}{\sigma_{2}} \operatorname{rot} \stackrel{2}{H} \cdot \bar{\tau}_{\alpha}
$$

we obtain

$$
I=-\sum_{\alpha=1}^{2} \int_{S_{0}} \mu_{1} \bar{\tau}_{\alpha} \times \bar{n} \cdot v \times \stackrel{1}{H} \psi \cdot \bar{\tau}_{\alpha} d S_{0}
$$

where we have used

$$
\psi \cdot \bar{\tau}_{\alpha}=\stackrel{1}{\psi} \cdot \bar{\tau}_{\alpha}=\stackrel{2}{\psi} \cdot \bar{\tau}_{\alpha}, \quad \alpha=1,2, \quad \text { on } S_{0} .
$$

Replacing the boundary term in (5.3) by 5.5 we derive the integral identity

$$
\begin{aligned}
\sum_{i=1}^{2} \int_{\substack{i \\
\Omega_{0}}}\left(\mu_{i} \stackrel{i}{H}_{t} \cdot \stackrel{i}{\psi}+\frac{1}{\sigma_{i}} \operatorname{rot}_{\xi} \stackrel{i}{H} \cdot \operatorname{rot}_{\xi} \stackrel{i}{\psi}\right) d \xi \\
=\sum_{i=1}^{2} \int_{\substack{i \\
\Omega_{0}}}^{i} f \cdot \stackrel{i}{f} d \xi+\sum_{\alpha=1}^{2} \int_{S_{0}} \mu_{1} \bar{\tau}_{\alpha} \times \bar{n} \cdot v \times \stackrel{1}{H} \psi \cdot \bar{\tau}_{\alpha} d S_{0} .
\end{aligned}
$$

To show existence of weak solutions to problem (5.1) satisfying the integral identity (5.6) we need the existence of a fundamental basis.

For this purpose we consider the elliptic problem

$$
\begin{aligned}
& \stackrel{1}{\mu}+\operatorname{rot}\left(\frac{1}{\sigma_{1}} \operatorname{rot} \stackrel{1}{\psi}\right)=\stackrel{1}{f}, \quad \operatorname{div} \stackrel{1}{\psi}=\stackrel{1}{h} \quad \text { in } \stackrel{1}{\Omega_{0}}, \\
& \stackrel{2}{\mu_{2}}+\operatorname{rot}\left(\frac{1}{\sigma_{2}} \operatorname{rot} \stackrel{2}{\psi}\right)=\stackrel{2}{f}, \quad \operatorname{div} \stackrel{2}{\psi}=\stackrel{2}{h} \quad \text { in } \stackrel{2}{\Omega_{0}}, \\
& \left(\frac{1}{\sigma_{1}} \operatorname{rot} \stackrel{1}{\psi}\right) \cdot \bar{\tau}_{\alpha}=\frac{1}{\sigma_{2}} \operatorname{rot} \stackrel{2}{\psi} \cdot \bar{\tau}_{\alpha}+g_{\alpha} \equiv g \cdot \bar{\tau}_{\alpha}, \quad \alpha=1,2, \quad \text { on } S_{0}, \\
& \stackrel{1}{\psi} \cdot \bar{n} \times \bar{\tau}_{\alpha}=\stackrel{2}{\psi} \cdot \bar{n} \times \bar{\tau}_{\alpha}, \quad \alpha=1,2, \quad \text { on } S_{0}, \\
& \stackrel{1}{\nu_{1} \psi} \cdot \bar{n}=\stackrel{2}{\nu} \nu_{2} \psi \bar{n} \\
& \text { on } S_{0} \text {, } \\
& \stackrel{2}{\left.\psi\right|_{B}}=0 .
\end{aligned}
$$

From 5.7$]_{1,2}$ we have $\mu_{i} \operatorname{div} \stackrel{i}{\psi}=\operatorname{div} \stackrel{i}{f}, i=1,2$. Hence for $\stackrel{i}{f}$ divergence free we see that $\psi$ is also divergence free.

Moreover, we have the compatibility conditions $\stackrel{i}{h}=\frac{1}{\mu_{i}} \operatorname{div} \stackrel{i}{f}, i=1,2$.

To prove existence of solutions to problem (5.7) we use the partition of unity introduced in Section 2. In view of the Fredholm theorem we only need to derive an a priori estimate. Therefore, we need 
Lemma 5.1. Consider problem (5.7). Assume that $\stackrel{i}{f} \in L_{2}(\stackrel{i}{\Omega}), \stackrel{i}{h} \in$ $H^{1}(\stackrel{i}{\Omega}), i=1,2$. Assume that the transmission and boundary conditions are satisfied: 5.7$]_{3,4,5,6}$. Then there exists a solution $(\stackrel{1}{\psi}, \stackrel{2}{\psi})$ to problem 5.7 such that $\stackrel{i}{\psi} \in H^{2}(\stackrel{i}{\Omega}), i=1,2$, and we have the estimate

$$
\sum_{i=1}^{2}\|\underset{2, i}{i}\|_{2, \Omega}^{i} \leq c \sum_{i=1}^{2}\left(|f|_{2, \Omega}^{i}+\|h\|_{1, \Omega}^{i}\right) .
$$

Proof. We use the partition of unity introduced in Section 2. Let $\zeta^{(k)}$ be a cut-off function from the partition of unity. Set $u^{(k)}=u \zeta^{(k)}$.

Take $k \in \mathfrak{M}_{1}$. Localization of 5.7 to $\operatorname{supp} \zeta^{(k)}$ implies that the problem can be treated as a problem in the whole $\mathbb{R}^{3}$ for functions with compact supports and then we apply the formula

$$
\operatorname{rot}^{2}=-\Delta+\nabla \operatorname{div} .
$$

In view of 5.9 problem 5.7 localized to $\operatorname{supp} \zeta^{(k)}, k \in \mathfrak{M}_{1}$, takes the form

$$
\begin{aligned}
& \mu_{1} \psi^{(k)}-\frac{1}{\sigma_{1}}\left(\Delta \dot{1}^{(k)}-\nabla \operatorname{div} \stackrel{1}{\psi}^{(k)}\right) \\
& =\frac{1}{\sigma_{1}}\left[-\Delta \stackrel{1}{\psi}^{(k)}+\nabla \operatorname{div} \stackrel{1}{\psi}^{(k)}-(-\Delta \stackrel{1}{\psi}+\nabla \operatorname{div} \stackrel{1}{\psi}) \zeta^{(k)}\right]+\stackrel{1}{f}^{(k)} \\
& =\frac{1}{\sigma_{1}}\left[-2{ }^{\prime} \stackrel{1}{\psi} \nabla \zeta^{(k)}-\stackrel{1}{\psi} \Delta \zeta^{(k)}+\operatorname{div} \stackrel{1}{\psi} \nabla \zeta^{(k)}+\nabla \stackrel{1}{\psi} \cdot \nabla \zeta^{(k)}+\stackrel{1}{\psi} \cdot \nabla \nabla \zeta^{(k)}\right] \\
& +\stackrel{1}{f^{(k)}} \equiv \stackrel{1}{f_{1}^{(k)}} \text {. }
\end{aligned}
$$

Moreover,

$$
\operatorname{div} \stackrel{1}{\psi}(k)=\stackrel{1}{\psi} \cdot \nabla \zeta^{(k)}+\stackrel{1}{h}{ }^{(k)} .
$$

Employing (5.11) in 5.10) yields

$$
\stackrel{1}{\mu_{1} \psi^{(k)}}-\frac{1}{\sigma_{1}} \Delta \stackrel{1}{\psi}^{(k)}=-\frac{1}{\sigma_{1}} \nabla\left(\stackrel{1}{\psi} \cdot \nabla \zeta^{(k)}+\stackrel{1}{h}^{(k)}\right)+\stackrel{1}{f_{1}^{(k)}} \equiv \stackrel{1}{f_{2}^{(k)}} .
$$

Multiplying 5.12 by ${ }^{(k)}$ and integrating over $\mathbb{R}^{3}$ gives

$$
\mu_{1}\left|\psi^{(k)}\right|_{2, \mathbb{R}^{3}}^{2}+\frac{1}{\sigma_{1}}\left|\nabla \psi^{(k)}\right|_{2, \mathbb{R}^{3}}^{2} \leq c\left|f_{2}^{(k)}\right|_{2, \mathbb{R}^{3}}\left|\psi^{(k)}\right|_{2, \mathbb{R}^{3}} .
$$

By the Young inequality we get

$$
\left\|\psi^{(k)}\right\|_{1, \mathbb{R}^{3}} \leq c\left|f_{2}^{(k)}\right|_{2, \mathbb{R}^{3}}
$$

Multiplying 5.12 by $\Delta \psi^{(k)}$ and integrating over $\mathbb{R}^{3}$ implies the estimate

$$
\left|\nabla \psi^{(k)}\right|_{2, \mathbb{R}^{3}}^{2}+\left|\Delta \psi^{(k)}\right|_{2, \mathbb{R}^{3}}^{2} \leq c\left|f_{2}^{(k)}\right|_{2, \mathbb{R}^{3}}^{2} .
$$


Hence, (5.14) and 5.15) imply

$$
\left\|\psi^{(k)}\right\|_{2, \mathbb{R}^{3}} \leq c\left|f_{2}^{(k)}\right|_{2, \mathbb{R}^{3}} .
$$

Similarly, for $k \in \mathfrak{M}_{2}$ we obtain

$$
\left\|\stackrel{2}{\psi}^{(k)}\right\|_{2, \mathbb{R}^{3}} \leq\left. c||_{2}^{(k)}\right|_{2, \mathbb{R}^{3}},
$$

where

$$
\begin{aligned}
& \stackrel{2}{f}_{2}^{(k)}=-\frac{1}{\sigma_{2}} \nabla\left(\stackrel{2}{\psi} \cdot \nabla \zeta^{(k)}+\stackrel{2}{h}^{(k)}\right)+\stackrel{2}{f}_{1}^{(k)}, \\
& \stackrel{2}{f_{1}^{(k)}}=\frac{1}{\sigma_{2}}\left[-2 \nabla \stackrel{2}{\psi} \nabla \zeta^{(k)}-\stackrel{2}{\psi} \Delta \zeta^{(k)}+\operatorname{div} \stackrel{2}{\psi} \nabla \zeta^{(k)}\right. \\
& \left.+\nabla \stackrel{2}{\psi} \cdot \nabla \zeta^{(k)}+\stackrel{2}{\psi} \cdot \nabla \nabla \zeta^{(k)}\right]+\stackrel{2}{f^{(k)}} .
\end{aligned}
$$

Let now $k \in \mathfrak{N}_{1}$. Then the localized problem (5.7) takes the form

$$
\begin{aligned}
& \mu_{i} \psi^{(k)}+\frac{1}{\sigma_{i}} \operatorname{rot}^{2} \stackrel{i}{\psi}{ }^{(k)}=\frac{1}{\sigma_{i}}\left(\operatorname{rot}^{2}{ }^{i} \psi^{(k)}-\operatorname{rot}^{2} \stackrel{i}{\psi} \zeta^{(k)}\right)+\stackrel{i}{f^{(k)}} \equiv \stackrel{i}{f_{1}^{(k)}}, \quad i=1,2, \\
& \operatorname{div} \stackrel{i}{\psi}^{(k)}=\stackrel{i}{\psi} \cdot \nabla \zeta^{(k)}+\stackrel{i}{h}(k) \equiv \stackrel{i}{h}{ }_{1}^{(k)}, \\
& \frac{1}{\sigma_{1}} \operatorname{rot} \stackrel{1}{\psi}^{(k)} \cdot \bar{\tau}_{\alpha}-\frac{1}{\sigma_{2}} \operatorname{rot}^{2} \psi^{(k)} \cdot \bar{\tau}_{\alpha}=\frac{1}{\sigma_{1}}\left(\operatorname{rot} \psi^{(k)}-\operatorname{rot}{ }^{1} \zeta^{(k)}\right) \cdot \bar{\tau}_{\alpha} \\
& -\frac{1}{\sigma_{2}}\left(\operatorname{rot} \stackrel{2}{\psi}(k)-\operatorname{rot} \stackrel{2}{\psi} \cdot \zeta^{(k)}\right) \cdot \bar{\tau}_{\alpha}+g_{\alpha}^{(k)} \equiv g_{\alpha 1}^{(k)}, \\
& \stackrel{1}{\psi}^{(k)} \cdot \bar{n} \times \bar{\tau}_{\alpha}=\stackrel{2}{\psi^{(k)}} \cdot \bar{n} \times \bar{\tau}_{\alpha}, \\
& i=1,2 \text {, }
\end{aligned}
$$

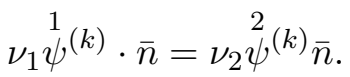

To examine problem (5.18) we pass to local coordinates which make the part of the boundary $S_{0} \cap \operatorname{supp} \zeta^{(k)} \equiv S_{0}^{(k)}$ flat. Let $\xi^{(k)}$ be the middle point of $S_{0}^{(k)}$. We introduce new local coordinates $y=\left(y_{1}, y_{2}, y_{3}\right)$ with origin at $\xi^{(k)}$ and such that $S_{0}^{(k)}$ is described by the equation

$$
y_{3}=F_{k}\left(y_{1}, y_{2}\right),
$$

where the point $\left(y_{1}, y_{2}, y_{3}\right)$, with $y_{3}>F_{k}\left(y_{1}, y_{2}\right)$ belongs to $\stackrel{1}{\Omega_{0}}$ and $\left(y_{1}, y_{2}, y_{3}\right)$ with $y_{3}<F_{k}\left(y_{1}, y_{2}\right)$ belongs to $\stackrel{2}{\Omega_{0}}$.

The coordinates $y=\left(y_{1}, y_{2}, y_{3}\right)$ are obtained from the global coordinates $x=\left(x_{1}, x_{2}, x_{3}\right)$ by a translation and a rotation. We denote this transformation by $y=Y_{k}(x)$. Next, we introduce new coordinates $z=\left(z_{1}, z_{2}, z_{3}\right)$ by 
the relations

$$
\begin{aligned}
& z_{\alpha}=y_{\alpha}, \quad \alpha=1,2, \\
& z_{3}=y_{3}-F_{k}\left(y_{1}, y_{2}\right) .
\end{aligned}
$$

We describe $(5.19)$ by $z=\Phi_{k}(y)=\Phi_{k}\left(Y_{k}(x)\right) \equiv \Psi_{k}(x)$. Hence, $S_{0}^{(k)}$ in coordinates $(5.19)$ is described by

$$
z_{3}=0 .
$$

Let $\chi=\chi(x)$ be any function. Set $\hat{\chi}^{(k)}(z)=\chi\left(\Psi_{k}^{-1}(z)\right)$ and $\tilde{\chi}^{(k)}(z)=$ $\hat{\chi}^{(k)}(z) \hat{\zeta}^{(k)}(z)$. Moreover, we define transformed operators by

$$
\hat{\nabla}_{z}=\left.\frac{\partial z}{\partial x}\right|_{x=\Psi_{k}^{-1}(z)} \nabla_{z} \text {. }
$$

Applying this transformation to problem (5.18) yields

$$
\begin{array}{ll}
\mu_{i} \tilde{\psi}^{(k)}+\frac{1}{\sigma_{i}} \hat{\operatorname{rot}}_{z}^{2} \tilde{\psi}^{(k)}=\tilde{f}_{1}^{(k)}, & i=1,2, \\
\operatorname{div}_{z} \tilde{\psi}^{(k)}=\tilde{h}_{1}^{(k)}, & i=1,2, \\
\frac{1}{\sigma_{1}} \hat{\operatorname{rot}}_{z} \tilde{\psi}^{(k)} \cdot \hat{\bar{\tau}}_{\alpha}-\frac{1}{\sigma_{2}} \hat{\operatorname{rot}}_{z} \tilde{\psi}^{(k)} \cdot \hat{\bar{\tau}}_{\alpha}=\tilde{g}_{\alpha 1}^{(k)}, & \alpha=1,2, \\
\tilde{\psi}^{(k)} \cdot \hat{\bar{n}} \times \hat{\bar{\tau}}_{\alpha}=\tilde{\tilde{\psi}}^{(k)} \cdot \hat{\bar{n}} \times \hat{\bar{\tau}}_{\alpha}, & \alpha=1,2, \\
\nu_{1} \tilde{\psi}^{(k)} \cdot \hat{\bar{n}}=\nu_{2} \tilde{\psi}^{(k)} \cdot \hat{\bar{n}}, &
\end{array}
$$

where

$$
\begin{aligned}
\hat{\bar{n}} & =\left(\begin{array}{c}
-F_{k, y_{1}} \\
-F_{k, y_{2}} \\
1
\end{array}\right) \frac{1}{\sqrt{1+F_{k, y^{\prime}}^{2}}}, \\
\hat{\bar{\tau}}_{1} & =\left(\begin{array}{c}
1 \\
0 \\
F_{k, y_{1}}
\end{array}\right) \frac{1}{\sqrt{1+F_{k, y_{1}}^{2}}}, \quad \hat{\bar{\tau}}_{2}=\left(\begin{array}{c}
0 \\
1 \\
F_{k, y_{2}}
\end{array}\right) \frac{1}{\sqrt{1+F_{k, y_{2}}}} .
\end{aligned}
$$

In view of the transformation 5.19 and the fact that $S_{0}^{(k)} \subset\left\{z_{3}=0\right\}$ we introduce the following tangent and normal vectors to the plane $z_{3}=0: \bar{n}_{z}=$ $(0,0,1), \bar{\tau}_{z 1}=(1,0,0), \bar{\tau}_{z 2}=(0,1,0)$. Then problem 5.20$)$ is transformed to

$$
\begin{aligned}
& \mu_{1} \tilde{\psi}^{(k)}+\frac{1}{\sigma_{1}} \operatorname{rot}_{z}^{2} \underset{\tilde{\psi}^{(k)}}{1}=\frac{1}{\sigma_{1}}\left(\operatorname{rot}_{z}^{2}-\hat{\operatorname{rot}}_{z}^{2}\right) \stackrel{1}{\tilde{\psi}^{(k)}}+\stackrel{\tilde{f}}{1}^{(k)} \equiv \tilde{f}_{2}^{1}(k), \quad z_{3}>0, \\
& \operatorname{div}_{z} \tilde{\psi}^{(k)}=\left(\operatorname{div}_{z}-\operatorname{div}_{z}\right) \stackrel{1}{1} \tilde{\psi}^{(k)}+\tilde{\tilde{h}}_{1}^{(k)} \equiv \stackrel{\tilde{h}}{2}^{(k)}, \quad z_{3}>0, \\
& \mu_{2} \tilde{\psi}^{(k)}+\frac{1}{\sigma_{2}} \operatorname{rot}_{z}^{2} \underset{\tilde{\psi}^{(k)}}{\stackrel{2}{\sigma_{2}}}=\frac{1}{\sigma_{2}}\left(\operatorname{rot}_{z}^{2}-\operatorname{rot}_{z}^{2}\right) \stackrel{2}{\tilde{\psi}^{(k)}}+\stackrel{2}{\tilde{f}_{1}^{(k)}} \equiv \stackrel{2}{\tilde{f}_{2}^{(k)}}, \quad z_{3}<0,
\end{aligned}
$$


[cont.]

$$
\begin{aligned}
& \operatorname{div}_{z} \stackrel{2}{\tilde{\psi}}^{(k)}=\left(\operatorname{div}_{z}-\hat{\operatorname{div}}_{z}\right) \stackrel{2}{\tilde{\psi}^{(k)}}+\stackrel{2}{h}_{1}^{(k)} \equiv \stackrel{2}{\tilde{h}}_{2}^{(k)}, \\
& z_{3}<0 \\
& \frac{1}{\sigma_{1}} \operatorname{rot}_{z} \tilde{\psi}^{(k)} \cdot \bar{\tau}_{2 \alpha}-\frac{1}{\sigma_{2}} \operatorname{rot}_{z} \stackrel{2}{\psi}^{(k)} \cdot \bar{\tau}_{2 \alpha} \\
& =\frac{1}{\sigma_{1}}\left(\operatorname{rot}_{z} \tilde{\psi}^{(k)} \cdot \bar{\tau}_{z \alpha}-\hat{\operatorname{rot}}_{z} \tilde{\psi}^{(k)} \cdot \hat{\bar{\tau}}_{\alpha}\right) \\
& -\frac{1}{\sigma_{2}}\left(\operatorname{rot}_{z} \stackrel{2}{\psi}^{(k)} \cdot \bar{\tau}_{z \alpha}-\hat{\operatorname{rot}}_{z} \tilde{\tilde{\psi}}^{(k)} \cdot \hat{\bar{\tau}}_{\alpha}\right)+\tilde{g}_{\alpha 1}^{(k)} \equiv \tilde{g}_{\alpha 2}^{(k)}, \quad \alpha=1,2, \quad z_{3}=0, \\
& \stackrel{1}{\tilde{\psi}^{(k)}} \cdot \bar{n}_{z} \times \bar{\tau}_{z \alpha}-\tilde{\psi}^{(k)} \cdot \bar{n}_{z} \times \bar{\tau}_{z \alpha}=\stackrel{1}{1} \tilde{\psi}^{(k)} \cdot\left(\bar{n}_{z} \times \bar{\tau}_{z \alpha}-\hat{\bar{n}} \times \hat{\bar{\tau}}_{\alpha}\right)
\end{aligned}
$$$$
-\tilde{\psi}^{(k)} \cdot\left(\bar{n}_{z} \times \bar{\tau}_{z \alpha}-\hat{\bar{n}} \times \hat{\bar{\tau}}_{z \alpha}\right) \equiv \tilde{b}_{\alpha}^{(k)}, \quad \alpha=1,2,
$$$$
z_{3}=0
$$$$
\nu_{1}^{1} \tilde{\psi}^{(k)} \cdot \bar{n}_{z}-\nu_{2} \tilde{\psi}^{(k)} \cdot \bar{n}_{z}
$$$$
=\stackrel{1}{\stackrel{1}{1}}(k) \cdot \stackrel{2}{\tilde{\psi}^{(k)}} \cdot\left(\bar{n}_{z}-\hat{\bar{n}}\right)-\nu_{2}{ }^{(k)} \cdot\left(\bar{n}_{z}-\hat{\bar{n}}\right) \equiv \tilde{a}^{(k)},
$$$$
z_{3}=0 \text {. }
$$

Since $k$ is fixed, we simplify problem 5.21 by writing it in the form

$$
\begin{aligned}
& \stackrel{1}{\mu_{1}} \underset{\psi}{\psi}+\frac{1}{\sigma_{1}} \operatorname{rot}^{2} \stackrel{1}{\psi}=\stackrel{1}{=} f_{2}, \\
& z_{3}>0 \\
& \operatorname{div} \stackrel{1}{\psi}=\stackrel{1}{h}{ }_{2}, \\
& z_{3}>0 \\
& \stackrel{2}{\mu_{2}} \psi+\frac{1}{\sigma_{2}} \operatorname{rot}^{2} \stackrel{2}{\psi}=\stackrel{2}{f_{2}}, \\
& z_{3}<0 \\
& \operatorname{div} \stackrel{2}{\psi}=\stackrel{2}{h}, \\
& z_{3}<0 \\
& \frac{1}{\sigma_{1}} \operatorname{rot} \stackrel{1}{\psi} \cdot \bar{\tau}_{z \alpha}-\frac{1}{\sigma_{2}} \operatorname{rot} \stackrel{2}{\psi} \cdot \bar{\tau}_{z \alpha}=g_{\alpha 2}, \quad \alpha=1,2, \quad z_{3}=0, \\
& \stackrel{1}{\psi} \cdot \bar{n}_{z} \times \bar{\tau}_{z \alpha}-\stackrel{2}{\psi} \cdot \bar{n}_{z} \times \bar{\tau}_{z \alpha}=b_{\alpha}, \quad \alpha=1,2, \quad z_{3}=0,
\end{aligned}
$$

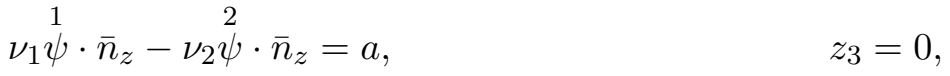

where all operators are with respect to the coordinates $z=\left(z_{1}, z_{2}, z_{3}\right)$, so the subscript $z$ is dropped for simplicity.

By employing formula 5.9 and the form of the tangent and normal vectors:

$$
\begin{aligned}
& \bar{n}_{z}=(0,0,1), \quad \bar{\tau}_{z 1}=(1,0,0), \quad \bar{\tau}_{z 2}=(0,1,0), \\
& \bar{n}_{z} \times \bar{\tau}_{z 1}=-\bar{\tau}_{z 2}, \quad \bar{n}_{z} \times \bar{\tau}_{z 2}=-\bar{\tau}_{z 1},
\end{aligned}
$$


problem 5.22 takes the form

$$
\begin{aligned}
& \stackrel{1}{\mu_{1}} \psi+\frac{1}{\sigma_{1}}(-\Delta \stackrel{1}{\psi}+\nabla \operatorname{div} \stackrel{1}{\psi})=\stackrel{1}{f_{2},} \quad z_{3}>0, \\
& \operatorname{div} \stackrel{1}{\psi}=\stackrel{1}{h}, \quad z_{3}>0, \\
& \stackrel{2}{\mu_{2}}+\frac{1}{\sigma_{2}}(-\Delta \stackrel{2}{\psi}+\nabla \operatorname{div} \stackrel{2}{\psi})=\stackrel{2}{f_{2},} \quad z_{3}<0, \\
& \operatorname{div} \stackrel{2}{\psi}=\stackrel{2}{h}, \quad z_{3}<0, \\
& \frac{1}{\sigma_{1}}\left(\stackrel{1}{\psi_{2, z_{3}}}-\stackrel{1}{\psi}{ }_{3, z_{2}}\right)-\frac{1}{\sigma_{2}}\left(\stackrel{2}{\psi_{2, z_{3}}}-\stackrel{2}{\psi_{3, z_{2}}}\right)=-g_{12}, \quad z_{3}=0, \\
& \frac{1}{\sigma_{1}}\left(\stackrel{1}{\psi_{1, z_{3}}}-\stackrel{1}{\psi_{3, z_{1}}}\right)-\frac{1}{\sigma_{2}}\left(\stackrel{2}{\psi_{1, z_{3}}}-\stackrel{2}{\psi_{3, z_{1}}}\right)=g_{22}, \quad z_{3}=0 \text {, } \\
& \stackrel{1}{\psi}{ }_{2}-\stackrel{2}{\psi}{ }_{2}=-b_{1} \text {, } \\
& z_{3}=0, \\
& \stackrel{1}{\psi_{1}}-\stackrel{2}{\psi_{1}}=-b_{2}, \\
& z_{3}=0, \\
& \stackrel{1}{\nu_{1} \psi_{3}}-\stackrel{2}{\nu_{2}} \psi_{3}=a, \\
& z_{3}=0 .
\end{aligned}
$$

Multiplying $5.23{ }_{1}$ by $\left.\nu_{1} \psi, \sqrt{4.23}\right)_{3}$ by $\stackrel{2}{\nu_{2} \psi}$, integrating over $\mathbb{R}_{+}^{3}$ and $\mathbb{R}_{-}^{3}$, respectively, and summing yields

$$
\begin{aligned}
& \nu_{1} \mu_{1}|\stackrel{1}{\psi}|_{2, \mathbb{R}_{+}^{3}}^{2}+\nu_{2} \mu_{2}|\stackrel{2}{\psi}|_{2, \mathbb{R}_{-}^{3}}^{2}+\frac{\nu_{1}}{\sigma_{1}} \int_{\mathbb{R}_{+}^{3}}(-\Delta \stackrel{1}{\psi}+\nabla \operatorname{div} \stackrel{1}{\psi}) \cdot \stackrel{1}{\psi} d z \\
& +\frac{\nu_{2}}{\sigma_{2}} \int_{\mathbb{R}_{-}^{3}}(-\Delta \stackrel{2}{\psi}+\nabla \operatorname{div} \stackrel{2}{\psi}) \cdot \stackrel{2}{\psi} d z=\nu_{1} \int_{\mathbb{R}_{+}^{3}} f_{2}^{1} \cdot \stackrel{1}{\psi} d z+\nu_{2} \int_{\mathbb{R}_{-}^{3}} f_{2}^{2} \cdot \stackrel{2}{\psi} d z .
\end{aligned}
$$

Integrating by parts the sum of the last two terms on the l.h.s. of (5.24) yields

$$
\begin{aligned}
& \frac{\nu_{1}}{\sigma_{1}} \int_{\mathbb{R}_{+}^{3}}[-\operatorname{div}(\nabla / \stackrel{1}{\psi} \cdot \stackrel{1}{\psi})+\operatorname{div}(\operatorname{div} \stackrel{1}{\psi} \psi)] d z+\frac{\nu_{1}}{\sigma_{1}} \int_{\mathbb{R}_{+}^{3}}\left(|\nabla \psi|^{2}-|\operatorname{div} \psi|^{2}\right) d z
\end{aligned}
$$

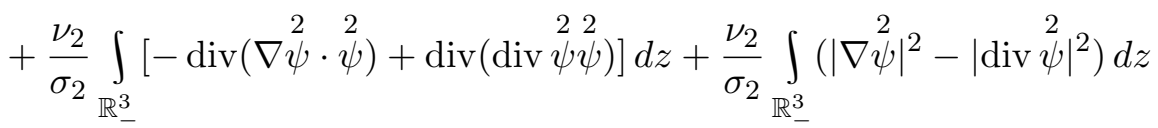

$$
\begin{aligned}
& =\frac{\nu_{1}}{\sigma_{1}}\left(|\nabla \psi|_{2, \mathbb{R}_{+}^{3}}^{2}-|\operatorname{div} \stackrel{1}{\psi}|_{2, \mathbb{R}_{+}^{3}}^{2}\right)+\frac{\nu_{1}}{\sigma_{2}}\left(|\nabla \psi|_{2, \mathbb{R}_{-}^{3}}^{2}-|\operatorname{div} \stackrel{2}{\psi}|_{2, \mathbb{R}_{-}^{3}}^{2}\right) \\
& -\int_{\mathbb{R}^{2}}\left(\frac{\nu_{1}}{\sigma_{1}} \stackrel{1}{\psi}, z_{3} \cdot \stackrel{1}{\psi}-\frac{\nu_{1}}{\sigma_{1}} \operatorname{div} \stackrel{1}{\psi} \underset{\psi}{\psi}{ }_{3}-\frac{\nu_{1}}{\sigma_{2}} \stackrel{2}{\psi}, z_{3} \cdot \stackrel{2}{\psi}+\frac{\nu_{2}}{\sigma_{2}} \operatorname{div} \stackrel{2}{\psi} \underset{\psi}{\psi}{ }_{3}\right) d z^{\prime},
\end{aligned}
$$


where $d z^{\prime}=d z_{1} d z_{2}$. Expressing the boundary term in 5.25 more explicitly, we have

$$
\begin{aligned}
& I=
\end{aligned}
$$

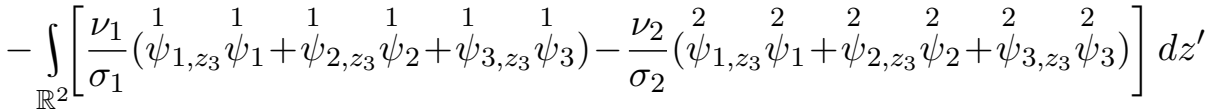

$$
\begin{aligned}
& \left.+\int_{\mathbb{R}^{2}}\left[\frac{\nu_{1}}{\sigma_{1}}\left(\stackrel{1}{\psi_{1, z_{1}}}+\stackrel{1}{\psi_{2, z_{2}}}+\stackrel{1}{\psi_{3, z_{3}}}\right) \stackrel{1}{\psi_{3}}-\frac{\nu_{2}}{\sigma_{2}} \stackrel{2}{\psi_{1, z_{1}}}+\stackrel{2}{\psi_{2, z_{2}}}+\stackrel{2}{\psi_{3, z_{3}}}\right) \stackrel{2}{\psi_{3}}\right] d z^{\prime} .
\end{aligned}
$$

Simplifying yields

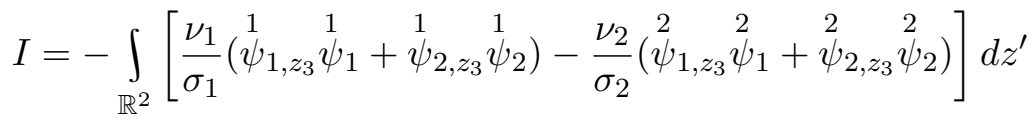

$$
\begin{aligned}
& +\int_{\mathbb{R}^{2}}\left[\frac{\nu_{1}}{\sigma_{1}}\left(\stackrel{1}{\psi_{1, z_{1}}}+\stackrel{1}{\psi_{2, z_{2}}}\right) \stackrel{1}{\psi_{3}}-\frac{\nu_{2}}{\sigma_{2}}\left(\stackrel{2}{\psi_{1, z_{1}}}+\stackrel{2}{\psi_{2, z_{2}}}\right) \stackrel{2}{\psi_{3}}\right] d z^{\prime} .
\end{aligned}
$$

If

$$
\frac{\nu_{1}}{\sigma_{1}} \stackrel{1}{\psi_{3}}=\frac{\nu_{2}}{\sigma_{2}} \stackrel{2}{\psi_{3}}+a
$$

the second term in $I$ equals

$$
\begin{aligned}
\int_{\mathbb{R}^{2}}\left[\left({ }^{1} \psi_{1, z_{1}}+\stackrel{1}{\psi_{2, z_{2}}}\right)\left(\frac{\nu_{2}}{\sigma_{2}} \stackrel{2}{\psi}_{3}+a\right)-\left(\stackrel{2}{\psi_{1, z_{1}}}+\stackrel{2}{\psi_{2, z_{2}}}\right) \frac{\nu_{2}}{\sigma_{2}} \psi_{3}\right] d z^{\prime} \\
=\int_{\mathbb{R}^{2}}\left(\begin{array}{c}
1 \\
\psi_{1, z_{1}}
\end{array} \stackrel{1}{\psi_{2, z_{2}}}\right) a d z^{\prime}
\end{aligned}
$$

If we assume the conditions

$$
\begin{aligned}
& \frac{\nu_{1}}{\sigma_{1}} \stackrel{1}{\psi}_{1, z_{3}}=\frac{\nu_{2}}{\sigma_{2}} \stackrel{2}{\psi_{1, z_{3}}}+\frac{\nu_{1}}{\sigma_{1}} \stackrel{1}{\psi_{3, z_{1}}}-\frac{\nu_{2}}{\sigma_{2}} \stackrel{2}{\psi}_{3, z_{1}}+g_{22}=a_{, z_{1}}+g_{22}, \\
& \frac{\nu_{1}}{\sigma_{1}} \stackrel{1}{\psi}_{2, z_{3}}=\frac{\nu_{2}}{\sigma_{2}} \stackrel{2}{\psi_{2, z_{3}}}+\frac{\nu_{1}}{\sigma_{1}} \stackrel{1}{\psi_{3, z_{2}}}-\frac{\nu_{2}}{\sigma_{2}} \stackrel{2}{\psi_{3, z_{2}}+g_{12}}=a_{, z_{2}}+g_{12},
\end{aligned}
$$

the first term in $I$ takes the form

$$
\begin{aligned}
& -\int_{\mathbb{R}^{2}}\left[\left(\frac{\nu_{2}}{\sigma_{2}} \stackrel{2}{\psi}_{1, z_{3}}+a_{, z_{1}}+g_{22}\right) \stackrel{1}{\psi_{1}}+\left(\frac{\nu_{2}}{\sigma_{2}} \stackrel{2}{\psi}_{2, z_{3}}+a_{, z_{2}}+g_{12}\right) \stackrel{1}{\psi_{2}}\right. \\
& \left.-\frac{\nu_{2}}{\sigma_{2}} \stackrel{2}{\psi_{1, z_{3}}}{ }^{2} \psi_{1}-\frac{\nu_{2}}{\sigma_{2}} \stackrel{2}{\psi_{2, z_{3}}} \stackrel{2}{\psi}_{2}\right] d z^{\prime} \\
& =-\int_{\mathbb{R}^{2}}\left[\left(a_{, z_{1}}+g_{22}\right) \psi_{1}^{1}+\left(a_{, z_{2}}+g_{12}\right) \psi_{2}^{1}\right] d z^{\prime} \text {. }
\end{aligned}
$$


Applying the above considerations in (5.24) and restoring the index $k$ gives

$$
\begin{aligned}
& \left|\psi^{(k)}\right|_{2, \mathbb{R}_{+}^{3}}^{2}+\left|\psi^{(k)}\right|_{2, \mathbb{R}_{-}^{3}}^{2}+\left\|\psi^{(k)}\right\|_{1, \mathbb{R}_{+}^{3}}^{2}+\left\|\psi^{(k)}\right\|_{1, \mathbb{R}_{-}^{3}}^{2} \\
& \leq c\left(\left|\operatorname{div} \stackrel{1}{\psi}^{(k)}\right|_{2, \mathbb{R}_{+}^{3}}^{2}+\left|\operatorname{div} \stackrel{2}{\psi^{(k)}}\right|_{2, \mathbb{R}_{-}^{3}}^{2}\right)+\left|\int_{\mathbb{R}^{2}}\left(\stackrel{1}{\psi}_{1, z_{1}}^{(k)}+\stackrel{2}{\psi}_{2, z_{2}}^{2}\right) a d z^{\prime}\right| \\
& +c\left|\int_{\mathbb{R}^{2}}\left[\left(a_{, z_{1}}^{(k)}+g_{22}^{(k)}\right) \stackrel{1}{\psi_{1}^{(k)}}+\left(a_{, z_{2}}^{(k)}+g_{12}^{(k)}\right){ }^{1} \psi_{2}^{(k)}\right] d z^{\prime}\right| \\
& +c\left|\int_{\mathbb{R}_{+}^{3}} f_{2}^{1(k)} \psi^{(k)} d z\right|+c\left|\int_{\mathbb{R}_{-}^{3}} f_{2}^{(k)} \psi^{2}(k) d z\right| .
\end{aligned}
$$

Next,

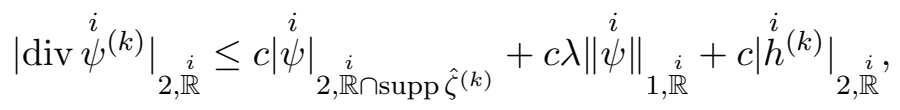

where $i=1,2, \stackrel{1}{\mathbb{R}}=\mathbb{R}_{+}^{3}, \stackrel{2}{\mathbb{R}}=\mathbb{R}_{-}^{3}$. Moreover,

$$
\begin{aligned}
& \left|\sum_{\alpha} \int_{\mathbb{R}^{2}} g_{\alpha 2}^{(k)} \frac{1}{\psi}(k) d z\right| \leq c \sum_{\alpha=1}^{2}\left|g_{\alpha 2}^{(k)}\right|_{2, \mathbb{R}^{2}}\left|\psi_{\alpha}^{(k)}\right|_{2, \mathbb{R}^{2}} \\
& \leq c \sum_{\alpha=1}^{2}\left[\left|\frac{1}{\psi}\right|_{2, \mathbb{R}^{2} \cap \operatorname{supp} \zeta^{(k)}}+\left|\psi^{2}\right|_{2, \mathbb{R}^{2} \cap \operatorname{supp} \zeta^{(k)}}+\lambda\left(\left|\nabla \psi^{(k)}\right|_{2, \mathbb{R}^{2}}+\left|\nabla \psi^{(k)}\right|_{\left.2, \mathbb{R}^{2}\right)}\right.\right. \\
& \left.\quad+\left|g_{\alpha}^{(k)}\right|_{2, \mathbb{R}^{2}}\right]\left|\psi_{\alpha}^{(k)}\right|_{2, \mathbb{R}^{2}} .
\end{aligned}
$$

Differentiate $5.23{ }_{1}$ and $5.233_{3}$ with respect to $\tau$, multiply by $\nu_{1} \psi_{, \tau}$ and 2 $\nu_{2} \psi_{, \tau}$, respectively, and add the second normal derivatives to get

$$
\begin{aligned}
& \sum_{i=1}^{2}\left\|\psi^{i}(k)\right\|_{2, \mathbb{R}}^{i} \\
& \quad \leq c \sum_{i=1}^{2}\left(\left\|\psi^{i}\right\|_{1, \mathbb{R} \cap \operatorname{supp} \zeta^{(k)}}^{i}+\lambda\left\|\psi^{(k)}\right\|_{2, \mathbb{R}}^{i}+\left|f^{(k)}\right|_{2, \mathbb{R}}^{i}+\left\|h^{i}(k)\right\|_{1, \mathbb{R}}\right) .
\end{aligned}
$$

Finally, we consider the case $k \in \mathfrak{N}_{2}$. Repeating the argument in the case $k \in \mathfrak{N}_{1}$ we obtain

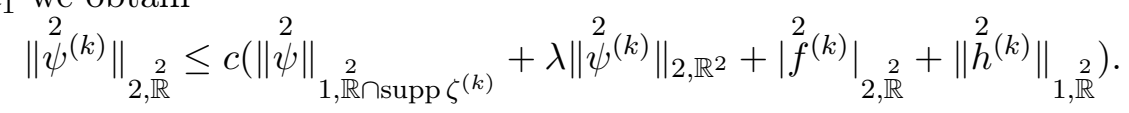

Employing inequalities (5.16), 5.17), 5.29), 5.30 and summing over all neighborhoods of the partition of unity we derive

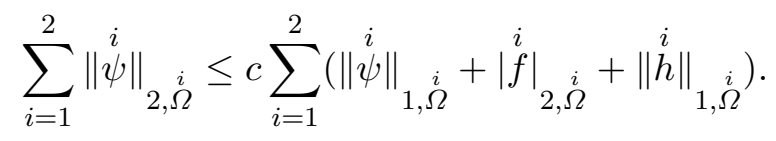


Hence, by interpolation we get

$$
\sum_{i=1}^{2}\|\stackrel{i}{i}\|_{2, \Omega}^{i} \leq c \sum_{i=1}^{2}\left(|\psi|_{2, \Omega}^{i}+|f|_{2, \Omega}^{i}+\|h\|_{1, \Omega}^{i}\right) .
$$

Multiply $(5.7)_{1,2}$ by $\stackrel{1}{\psi}, \stackrel{2}{\psi}$, respectively, integrate over $\stackrel{i}{\Omega}, i=1,2$, respectively and exploit the boundary and transmission conditions to get

$$
\sum_{i=1}^{2}\left|{ }^{i}\right|_{2, \Omega}^{i} \leq c \sum_{i=1}^{2}|f|_{2, \Omega}^{i} .
$$

Inequalities 5.32 and 5.33 imply

$$
\sum_{i=1}^{2} \underset{2 i}{i} \|_{2, \Omega}^{i} \leq c \sum_{i=1}^{2}\left(|f|_{2, \Omega}^{i}+\|h\|_{1, \Omega}^{i}\right) .
$$

This implies (5.8).

To conclude the proof we need existence of solutions to problem (5.7) (see [15, Ch. 4, Sect. 7]). For this purpose we have to examine the localized versions of problem 5.7) to the neighborhoods $\operatorname{supp} \zeta^{(k)}, k \in \mathfrak{M}_{1} \cup \mathfrak{M}_{2} \cup$ $\mathfrak{N}_{1} \cup \mathfrak{N}_{2}$. Therefore, we have problem (5.10) for $k \in \mathfrak{M}_{1}$, a problem similar to (5.10) for $k \in \mathfrak{M}_{2}$, problem (5.22) for $k \in \mathfrak{N}_{1}$ and finally the localized problem for $k \in \mathfrak{N}_{2}$. Assume that $h$ denotes all r.h.s. functions of these problems. Since all the localized linear problems are solvable, there exists an operator $R$, called a regularizer, such that

$$
R h=\sum_{k} \sum_{i=1}^{2} \eta^{(k)} \stackrel{i}{\psi}^{(k)},
$$

where $k \in \mathfrak{M}_{1} \cup \mathfrak{M}_{2} \cup \mathfrak{N}_{1} \cup \mathfrak{N}_{2}$.

Let $A$ be the operator of problem (5.5). The calculations imply existence of operators $T$ and $W$ such that

$$
A R h=h+T h \quad \text { and } \quad R A \psi=\psi+W \psi,
$$

where $\psi=(\stackrel{1}{\psi}, \stackrel{2}{\psi})$. For sufficiently small $\lambda$ and large $\left(\mu_{1}, \mu_{2}\right)$ we have $\|T\|,\|W\|<1$. This implies existence of solutions to problem (5.7) and ends the proof.

Consider the problem

$$
\begin{aligned}
& \Delta \stackrel{i}{\varphi}=\stackrel{i}{h} \quad \text { in } \stackrel{i}{\Omega_{0}}, i=1,2, \\
& \stackrel{1}{\varphi}=\stackrel{2}{\varphi}, \quad \mu_{1} \bar{n} \cdot \nabla \stackrel{1}{\varphi}=\mu_{2} \bar{n} \cdot \nabla^{2} \quad \text { on } S_{0}, \\
& \left.\bar{n} \cdot \nabla \stackrel{2}{\varphi}\right|_{B}=0, \\
& \int \stackrel{i}{\varphi} d x=0, i=1,2 \text {. } \\
& \stackrel{i}{\Omega_{0}}
\end{aligned}
$$


Lemma 5.2. Assume that $\stackrel{i}{h} \in \stackrel{i}{\Omega_{t}}, i=1,2$. Then there exists a solution to problem (5.35) such that $\stackrel{i}{\varphi} \in H^{2}\left(\stackrel{i}{\Omega_{t}}\right), i=1,2$, and we have the estimate

$$
\sum_{i=1}^{2}\|\stackrel{i}{\varphi}\|_{H^{2}\left(\Omega_{0}\right)}^{i} \leq c \sum_{i=1}^{2}\|\stackrel{i}{h}\|_{L_{2}\left(\Omega_{0}\right)}^{i} .
$$

Proof. To show existence of weak solutions to problem 5.35 we find an energy estimate and apply the Fredholm theorem. Therefore, we multiply 5.35) 1 by $\stackrel{i}{\varphi}$ and integrate over $\stackrel{i}{\Omega}$. After summation we get

$$
\sum_{i=1}^{2} \int_{\substack{i \\ \Omega_{0}}} \mu_{i} \Delta \stackrel{i}{\varphi} \varphi^{i} d x=\sum_{i=1}^{2} \int_{\substack{i \\ \Omega_{0}}} \mu_{i}{ }^{i}{ }^{i} \varphi d x .
$$

Integration by parts yields

$$
\begin{aligned}
\sum_{i=1}^{2} \int_{\substack{i \\
\Omega_{0}}} \mu_{i}\left|\nabla \varphi^{i}\right|^{2} d x+\int_{S_{0}}\left(\mu_{1} \bar{n} \cdot \nabla \stackrel{1}{\varphi} \underset{\varphi}{1}-\mu_{2} \bar{n} \cdot \nabla \stackrel{2}{\varphi} \stackrel{2}{\varphi}\right) d S_{0} & \\
& =-\sum_{i=1}^{2} \int_{\substack{i \\
\Omega_{0}}} \mu_{i} \stackrel{i}{h} \underset{\varphi}{\varphi} d x .
\end{aligned}
$$

In view of 5.35$)_{2}$ the boundary term in (5.37) vanishes. Then 5.354 and the Poincaré inequality give

$$
\sum_{i=1}^{2}\|\stackrel{i}{\varphi}\|_{H^{1}\left(\Omega_{0}\right)} \leq c \sum_{i=1}^{2}\|\stackrel{i}{h}\|_{L_{2}\left(\Omega_{0}\right)}^{i} .
$$

Now the Fredholm theorem gives existence of weak solutions to problem 5.35 under the assumption that $\stackrel{i}{h} \in L_{2}\left(\stackrel{i}{\Omega_{t}}\right), i=1,2$.

Applying the technique of regularizer we improve regularity of the weak solutions and prove estimate (5.36). This concludes the proof.

Setting

$$
\stackrel{i}{v}=\stackrel{i}{\psi}-\nabla \stackrel{i}{\varphi}, \quad i=1,2
$$

we see that $\stackrel{1}{v}, \stackrel{2}{v}$ are solutions to the problem

$$
\begin{array}{ll}
\mu_{i} \stackrel{i}{v}+\operatorname{rot}\left(\frac{1}{\sigma_{i}} \operatorname{rot} \stackrel{i}{v}\right)=\stackrel{i}{f}-\mu_{i} \nabla_{\varphi}^{i}, & \text { in } \Omega_{0}, i=1,2, \\
\operatorname{div} \stackrel{i}{v}=0, & \\
\frac{1}{\sigma_{1}} \operatorname{rot} \stackrel{1}{v} \cdot \bar{\tau}_{\alpha}=\frac{1}{\sigma_{2}} \operatorname{rot} \stackrel{2}{v} \cdot \bar{\tau}_{\alpha}+g_{\alpha}, \quad \alpha=1,2, & \text { on } S_{0}, \\
\stackrel{1}{v} \cdot \bar{n} \times \bar{\tau}_{\alpha}=\stackrel{2}{v} \cdot \bar{n} \times \bar{\tau}_{\alpha}, \quad \alpha=1,2, & \text { on } S_{0},
\end{array}
$$




$$
\begin{aligned}
& \nu_{1} \cdot \stackrel{1}{v} \cdot \bar{n}=\nu_{2}^{2} \cdot \bar{n} \quad \text { on } S_{0}, \\
& \left.\stackrel{2}{v}\right|_{B}=0 .
\end{aligned}
$$

DEFINITION 5.3. Introduce a set of smooth divergence free functions $S^{k}\left(\Omega_{0}\right)=H^{k}\left(\stackrel{1}{\Omega_{0}}\right) \times H^{k}\left(\stackrel{2}{\Omega_{0}}\right)$ satisfying the transmission conditions $5.40{ }_{2,3,4}$ and the boundary condition 5.40$)_{5}$. Then problem (5.40) implies existence of an operator

$$
A: S^{2}\left(\Omega_{0}\right) \rightarrow S^{0}\left(\Omega_{0}\right) .
$$

In view of Lemma 5.1 the inverse operator

$$
A^{-1}: S^{0}\left(\Omega_{0}\right) \rightarrow S^{2}\left(\Omega_{0}\right) \subset S^{0}\left(\Omega_{0}\right)
$$

exists. Hence $A^{-1}$ is a compact operator

$$
A^{-1}: S^{0}\left(\Omega_{0}\right) \rightarrow S^{0}\left(\Omega_{0}\right) .
$$

Moreover, the operator $A$ is symmetric, because

$$
(A \psi, \varphi)=\sum_{i=1}^{2}\left[\mu_{i}(\stackrel{i}{\psi}, \stackrel{i}{\varphi})+\frac{1}{\sigma_{i}}(\operatorname{rot} \stackrel{i}{\psi}, \operatorname{rot} \stackrel{i}{\varphi})\right]=(\psi, A \varphi) .
$$

From the von Neumann theorem (see [17, Ch. 4, Sect. 3]) we know that $A^{-1}$ is also a symmetric operator. Since $A^{-1}$ is compact and symmetric, the Fredholm theorem (see [13, Ch. 1]) yields

THEOREM 5.4. The operator $A^{-1}$ has a countable set of eigenvalues. To each eigenvalue $\lambda$ corresponds at least one nontrivial solution to the equation

$$
\lambda \psi=A^{-1} \psi .
$$

The eigenfunctions to problem 5.41 form a dense set of functions in $S^{0}\left(\Omega_{0}\right)$. The functions can be chosen as basic functions for the FaedoGalerkin method.

Acknowledgements. The research leading to these results has received funding from the People Programme (Marie Curie Actions) of the European Union's Seventh Framework Programme FP7/2007-2013/ under REA grant agreement No. 319012 and from the Funds for International Co-operation under Polish Ministry of Science and Higher Education grant agreement No. 2853/7.PR/2013/2.

\section{References}

[1] E. B. Bykhovskiı̆, Solvability of the mixed problem for the Maxwell equations in the case of ideal conductive boundary, Vestnik Leningrad Univ. Ser. Mat. Mekh. Astronom. 13 (1957), no. 13, 50-66 (in Russian).

[2] G. H. A. Cole, Fluid Dynamics, Methuen, London \& Colchester, 1962. 
[3] E. Frolova, Free boundary problem of magnetohydrodynamics, Zap. Nauchn. Sem. POMI 425 (2014), 149-178 (in Russian).

[4] E. Frolova and V. A. Solonnikov, Solvability of a free boundary problem of magnetohydrodynamics in an infinite time interval, Zap. Nauchn. Sem. POMI 410 (2013), 131-167 (in Russian).

[5] P. Kacprzyk, Local free boundary problem for incompressible magnetohydrodynamics, Dissertationes Math. 509 (2015), 52 pp.

[6] P. Kacprzyk, Global free boundary problem for incompressible magnetohydrodynamics, Dissertationes Math. 510 (2015), 44 pp.

[7] P. Kacprzyk, Local existence of solutions of the free boundary problem for the equations of a magnetohydrodynamic incompressible fluid, Appl. Math. (Warsaw) 30 (2003), 461-488.

[8] P. Kacprzyk, Almost global solutions of the free boundary problem for the equations of a magnetohydrodynamic incompressible fluid, Appl. Math. (Warsaw) 31 (2004), 69-77.

[9] P. Kacprzyk, Free boundary problem for the equations of magnetohydrodynamic incompressible viscous fluid, Appl. Math. (Warsaw) 34 (2007), 75-95.

[10] P. Kacprzyk and W. M. Zajączkowski, On the Faedo-Galerkin method for a free boundary problem for incompressible viscous magnetohydrodynamics, Topol. Methods Nonlinear Anal. 52 (2018), 69-98.

[11] L. Kapitanskii and K. Pileckas, On some problems of vector analysis, Zap. Nauchn. Sem. LOMI 138 (1984), 65-85 (in Russian).

[12] N. E. Kochin, Vector Calculus and Introduction to Tensor Calculus, Izdat. Akad. Nauk SSSR, Moscow, 1951 (in Russian).

[13] O. A. Ladyzhenskaya, Boundary Value Problems of Mathematical Physics, Nauka, Moscow, 1973 (in Russian).

[14] O. A. Ladyzhenskaya and V. A. Solonnikov, Solvability of some nonstationary problems of magnetohydrodynamics for viscous incompressible fluids, Trudy Mat. Inst. Steklova 59 (1960), 115-173 (in Russian).

[15] O. A. Ladyzhenskaya, V. A. Solonnikov and N. N. Ural'tseva, Linear and Quasilinear Equations of Parabolic Type, Nauka, Moscow, 1967 (in Russian).

[16] L. D. Landau, E. M. Lifshitz and L. P. Pitaevskii, Electrodynamics of Continuous Media, 2nd ed., Landau and Lifshitz Course of Theoretical Physics, Vol. 8.

[17] K. Maurin, Methods of Hilbert Spaces, Państwowe Wydawnictwo Naukowe, Warszawa, 1959 (in Polish).

[18] M. Padula and V. A. Solonnikov, On the free boundary problem of mhd, Zap. Nauchn. Sem. POMI 385 (2010), 135-186 (in Russian).

[19] M. Sakhaev and V. A. Solonnikov, On some stationary problems of magnetohydrodynamics in general domains, Zap. Nauchn. Sem. POMI 397 (2011), 126-149 (in Russian).

[20] Y. Shibata and W. M. Zajączkowski, On local motion for a free boundary problem for incompressible viscous magnetohydrodynamics in the $L_{p}$-approach. Part 1 , submitted to Dissertationes Math.

[21] Y. Shibata and W. M. Zajacczkowski, On local motion for a free boundary problem for incompressible viscous magnetohydrodynamics in the $L_{p}$-approach. Part 2 , submitted to Dissertationes Math.

[22] V. A. Solonnikov, Estimates of solutions of an initial-boundary value problem for the linear non-stationary Navier-Stokes system, Zap. Nauchn. Sem. LOMI 59 (1976), 178-254 (in Russian). 
[23] V. A. Solonnikov, Estimates of solutions to nonstationary linearized Navier-Stokes system, Trudy Mat. Inst. Steklova 70 (1964), 213-317 (in Russian).

[24] V. A. Solonnikov, On an unsteady motion of an isolated volume of a viscous incompressible fluid, Izv. Akad. Nauk SSSR Ser. Mat. 51 (1987), 1065-1087 (in Russian).

[25] R. Temam, Navier-Stokes Equations: Theory and Numerical Analysis, Amer. Math. Soc., 2001.

[26] W. M. Zajączkowski, On nonstationary motion of a compressible barotropic viscous fluid bounded by a free surface, Dissertationes Math. 324 (1993), 101 pp.

Piotr Kacprzyk

Institute of Mathematics and Cryptology

Cybernetics Faculty

Military University of Technology

S. Kaliskiego 2

00-908 Warszawa, Poland

ORCID: 0000-0003-1504-5394

E-mail: pk_wat@wp.pl
Wojciech M. Zajączkowski

Institute of Mathematics

Polish Academy of Sciences

Śniadeckich 8

00-656 Warszawa, Poland

ORCID: 0000-0003-1229-2162

E-mail:wz@impan.pl 
